\title{
Variationally-based theories for buckling of partial composite beam-columns including shear and axial effects
}

\author{
Noël Challamel ${ }^{\mathrm{a}, *}$, Ulf Arne Girhammar ${ }^{\mathrm{b}, \mathrm{c}}$ \\ a Université Européenne de Bretagne, Laboratoire de Génie Civil et Génie Mécanique (LGCGM), INSA de Rennes, 20, avenue des Buttes de Coësmes, 35043 Rennes cedex, France

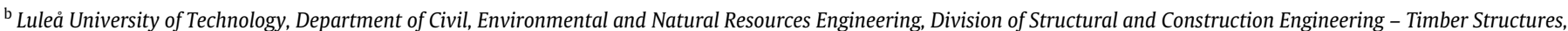 \\ SE-97187 Luleå, Sweden \\ ${ }^{\mathrm{c}}$ Umeå University, Faculty of Science and Technology, Department of TFE - Division of Building Engineering, SE-90187 Umeå, Sweden
}

\begin{abstract}
This paper is focused on elastic stability problems of partial composite columns: the conditions for the axial load not to introduce any pre-bending effects in composite columns; the equivalence, similarities and differences between different sandwich and partial composite beam theories with and without the effect of shear, with and without the effect of axial extensibility, and also the effect of eccentric axial load application. The basic modelling of the composite beam-column uses the Euler-Bernoulli beam theory and a linear constitutive law for the slip. In the analysis of this reference model, a variational formulation is used in order to derive relevant boundary conditions. The specific loading associated with no prebending effects before buckling is geometrically characterized, leading to analytical buckling loads of the partial composite column. The equivalence between the Hoff theory for sandwich beam-columns, the composite action theory for beam-columns with interlayer slip and the corresponding Bickford-Reddy theory, is shown from the stability point of view. Special loading configurations including eccentric axial load applications and axial loading only on one of the sub-elements of the composite beam-column are investigated and the similarity of the behaviour to that of imperfect ordinary beam-columns is demonstrated. The effect of axial extensibility on kinematical relationships (according to the Reissner theory), is analytically quantified and compared to the classical solution of the problem. Finally, the effect of incorporating shear in the analysis of composite members using the Timoshenko theory is evaluated. By using a variational formulation, the buckling behaviour of partial composite columns is analysed with respect to both the Engesser and the Haringx theory. A simplified uniform shear theory (assuming equal shear deformations in each sub-element) for the partial composite beam-column is first presented, and then a refined differential shear theory (assuming individual shear deformations in each sub-element) is evaluated. The paper concludes with a discussion on this shear effect, the differences between the shear theories presented and when the shear effect can be neglected.
\end{abstract}

\section{Background and problem formulation}

\subsection{Introduction and literature review}

This paper is devoted to the stability problem of a partial composite column, or equivalent composite sandwich column, and more specifically on the specific loading mode influencing the stability problem. Layered structural elements with interlayer slip are typically encountered in wood design, where wooden beams are made up of layers assembled by means of nailing,

\footnotetext{
* Corresponding author. Tel.: +33 02232384 78; fax: +33 0223238491 .

E-mail addresses: noel.challamel@insa-rennes.fr (N. Challamel), ulfarne.girhammar@tfe.umu.se (U.A. Girhammar).
}

bolting or gluing (with a soft shear modulus). Partial composite structures built up by sub-elements of different materials and connected by shear connectors to form an interacting unit, such as timber-concrete or steel-concrete elements, are widely used in building engineering. In the case of a flexible connection, the analysis procedure requires consideration of the interlayer slip between the sub-elements, leading to the partial interaction concept.

For a detailed literature background on the partial composite theory, the readers are referred to Girhammar and Gopu [1] and Girhammar and Pan [2]. Möhler [3] obtained the buckling formulae of axially loaded partial composite columns, using Euler-Bernoulli model for each sub-element of the beam-column. The lateral buckling problem of partial composite beam-columns subjected to both transverse and axial loading was investigated by e.g. Girhammar and Gopu [4,5] and Girhammar and Pan [2] 


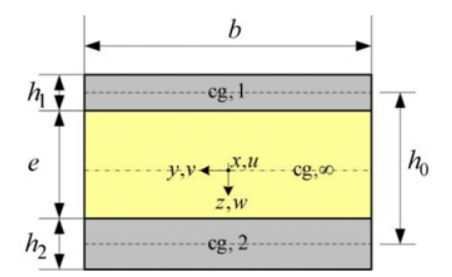

Fig. 1. Geometric parameters of a sandwich beam-column.

for general boundary conditions. Xu and $\mathrm{Wu}[6]$ generalized the results of Girhammar and Gopu [1] by using the Timoshenko beam theory based on Engesser theory. Xu and $\mathrm{Wu}$ [6] presented a partial composite shear beam model with uniform shear behaviour for each beam. More recently, Schnabl and Planinc [7] suggested to add an extensibility effect on the Euler-Bernoulli based solution of partial composite beam and showed the importance of the choice of boundary conditions for the buckling problem. Schnabl and Planinc [8] recently obtained some buckling solutions from the partial composite Reissner beam theory, leading to differential shear beam solution based on Haringx theory.

Sandwich beams are usually composed of three layers, two thin faces and a thick weak shear core [9-11]. The static in-plane behaviour of sandwich beams is well established by Hoff [12]. The in-plane buckling problem of a three-layer sandwich beam was studied by Hoff and Mautner [13] (see e.g. also [12,14,15]; see more recently $[16,17])$. A sixth-order differential equation is obtained for the deflection, and the similarity between this governing equation and that for composite beams with partial interaction was demonstrated for instance by Heuer [18]. Therefore, the paper is applied to both partial composite action and sandwich theories. Blaauwendraad [19] and Kardomateas [20] recently summarized shear and sandwich column buckling theory, and questioned the relevancy of each theory for structural design. There is a need to clarify the role of boundary conditions, extensibility and shear effects on the design of these structural members from an engineering point of view.

\subsection{Characteristics of the partial composite beam-column model}

\subsubsection{Geometry of the cross-section}

The state of the composite or sandwich beam (see Figs. 1 and 2 ) is specified by (i) the in-plane deflection $w(x)$ in the $x z$ plane, (ii) the axial displacement $u_{1}(x)$ of the beam axis of the domain " 1 " in the $x$ direction and (iii) the displacement $u_{2}(x)$ of the beam axis of the domain " 2 " in the $x$ direction. Each sub-domain is assumed to be composed of a beam of width $b_{i}$ and depth $h_{i}$. For sandwich beams, it is often assumed that the width of each beam is identical, i.e. $b_{i}$ is equal to $b$ (Fig. 1); $e$ is the depth of the interlayer. The geometric parameters defining a typical composite beam-column with two sub-elements of different geometry and materials are shown in Fig. 2. The subscripts ' 1 ' and ' 2 ' refer to the top and bottom elements of the cross-section, respectively. The displacements in the $x-, y$-, and $z$-direction are denoted $u, v$, and $w$, respectively. The origin of the coordinate axis is located in the centroid of the full composite section $(\mathrm{cg}, \infty)$.

\subsubsection{Shear interlayer and slip modulus}

The sub-elements are connected together by means of some kind of discrete shear connectors or a weak shear layer, which are assumed to produce uniformly distributed slip forces or interlayer shear stresses. The shear connector or shear layer force per unit length versus the slip or shear displacement behaviour is linear elastic with a constant slip modulus, $K$, with respect to bending in the transverse direction. The slip between the

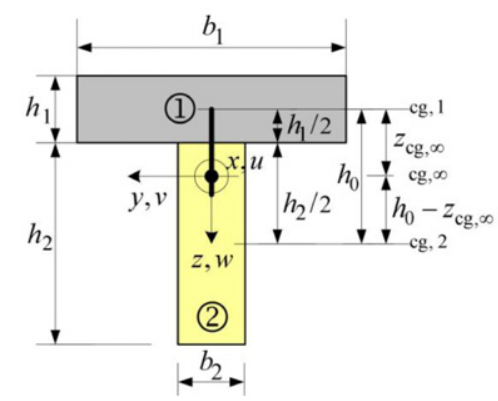

Fig. 2. Geometric parameters of a partial composite beam-column.

two sub-elements (Fig. 2) or the shear deformation of the core (Fig. 1) is denoted $\Delta u$. Frictional effects and uplift at the shear interface are neglected. It is postulated that the flexible connection will only be associated with interface shear interaction, without any normal interaction between the sub-elements. This last assumption can be justified, as shown, for instance, by Adekola [21] (see more recently [22-25]). There are a considerable number of experimental verifications in the literature with respect to the applicability of the classical composite action theory neglecting the effect of the vertical separation between each sub-element and assuming equal curvatures for the two sub-elements. In addition, for example in [26], nailed shear connections in composite timber structures are tested with respect to both slip and uplift and no significant effect of including the transverse relationship was found. If transverse partial interaction can be neglected, also the difference between the curvatures of the two sub-components can be neglected. Neglecting transverse separation will not affect the results obtained in this paper. Frictional effects are usually neglected because there is an uncertainty with respect to the magnitude that will be developed in real structures. It is usually on the safe side to neglect the frictional effects. It is customary to eliminate the frictional effects in experimental evaluation of the slip modulus, by using shims or some other frictionless materials between the sub-elements.

Full composite action (infinite slip modulus, $K \rightarrow \infty$ ) and noncomposite action (zero slip modulus, $K \rightarrow 0$ ) represent upper and lower bounds for the partial composite action, respectively.

It can be shown that there is an equivalence with respect to the fundamental behaviour and governing differential equations between shear connections (lap joints mechanically or adhesively jointed), sandwich constructions (sandwich beams and columns with cores of low shear rigidity) and composite structures with interlayer slip (see the references in [27]). The correspondence between the partial composite beam-columns (with slip modulus, $K$ ) and the three-layer sandwich beam (with the shear modulus, $G^{*}$ ) is immediate from the following identity (cf. Fig. 1):

$$
\frac{b G^{*}}{e}=K
$$

where $G^{*}$ is the shear modulus of the soft core, $e$ is the depth of the core or interlayer and $b$ the width of this core or shear interlayer.

It should be mentioned that the equivalence between the slip modulus of shear connectors and shear modulus of glue lines of finite thickness or sandwich-type of cores was first demonstrated by McCutcheon [28]. This equivalence is also discussed by Krawczyk et al. [29] for the buckling problem of laminated beam-columns, by Girhammar et al. [27] for the in-plane dynamics problem, and by Challamel [30] for the out-of-plane behaviour (see also [31]). With the substitution of $K$ according to Eq. (1), all results obtain in this study are also applicable to sandwich-type of beams with a shear modulus $G^{*}$ for the core. 


\subsubsection{Parameters of the cross-section and the partial interaction}

For convenience, some quantities and parameters for the partial composite beam-column and its cross-section, as shown in Fig. 2, are introduced (cf. $[5,2])$. Parameters including the slip modulus are

$\alpha^{2}=K\left(\frac{1}{E_{1} A_{1}}+\frac{1}{E_{2} A_{2}}+\frac{h_{0}^{2}}{E I_{0}}\right)$

$\beta=\frac{K h_{0}}{E I_{0}}$

and the axial and bending stiffnesses are given by

$E A_{0}=E_{1} A_{1}+E_{2} A_{2}$

$E A_{\mathrm{p}}^{2}=E_{1} A_{1} \cdot E_{2} A_{2}$

$E I_{0}=E_{1} I_{1}+E_{2} I_{2}$

$E I_{\infty}=E I_{0}\left(1+\frac{E A_{\mathrm{p}}^{2} \cdot h_{0}^{2}}{E A_{0} \cdot E I_{0}}\right)=E I_{0}\left(1-\frac{\beta h_{0}}{\alpha^{2}}\right)^{-1}$.

The subscripts 1 and 2 refer to respective sub-element and the subscripts 0 and $\infty$ denote the quantities in case of non-composite action and full composite action, respectively. It is noted that the expression $\beta h_{0} / \alpha^{2}$ is a non-dimensional parameter independent of $K$, that $\alpha L$, where $L$ is the length of the beam-column, is a nondimensional parameter including the effect of the slip modulus $K$, and that

$\frac{E I_{\infty}}{E I_{0}}=1+\frac{\frac{h_{0}^{2}}{E I_{0}}}{\frac{1}{E_{1} A_{1}}+\frac{1}{E_{2} A_{2}}}=\frac{1}{1-\frac{\beta h_{0}}{\alpha^{2}}}$

is a parameter representing the relative bending stiffness of the cross-section. The depth between the centroids of the two subelements is given by

$h_{0}=\frac{h_{1}+h_{2}}{2}$

and the location of the centroid of the full composite section by

$z_{\mathrm{cg}, \infty}=\frac{E_{2} A_{2}}{E A_{0}} h_{0}$.

\subsection{Different beam-column theories and pertaining kinematical assumptions}

\subsubsection{Ordinary solid beam-column theories}

There are a number of theories for ordinary solid beam-columns with different types of kinematical assumptions. These theories are classified in this section from a hierarchical point of view, from the Euler-Bernoulli beam-column theory to higher-order theories including shear deformations. We follow the presentation of Reddy [32]. Then, we extend this classification to composite beam-columns with partial interaction, composed of two independent sub-components connected to one another by shear connectors, where the slip forces or shear flow are represented by a simple connection model.

In the simplest beam bending model, the Euler-Bernoulli beam model, the only nonzero strain is:

$\varepsilon_{x x}=u^{\prime}-z w^{\prime \prime}=\varepsilon_{x x}^{0}+z \chi \quad$ where $\varepsilon_{x x}^{0}=u^{\prime}$ and $\chi=-w^{\prime \prime}$

where prime etc. denote differentiation with respect to $x, \varepsilon_{x x}^{0}$ is the extensional strain of the neutral axis, and $\chi$ is the bending curvature strain. The inextensible Euler-Bernoulli model is obtained by setting $\varepsilon_{x x}^{0}=0$. The buckling of inextensible Euler-Bernoulli based columns was first studied by Euler [33]. Euler [33] not only studied the linearized equations but also investigated the geometrically exact problem called the Elastica using elliptic integrals. The buckling of extensional Euler-Bernoulli columns has been investigated by Pflüger [34] (see also [35-37]). In the hierarchical classification, the Timoshenko model can be considered as a superior model corresponding to the Euler-Bernoulli model, but extended to include the shear effect. The kinematics of the Timoshenko model can be presented in the following format:

$$
\begin{gathered}
\varepsilon_{x x}=u^{\prime}-z \psi^{\prime}=\varepsilon_{x x}^{0}+z \chi \text { and } 2 \varepsilon_{x z}=w^{\prime}-\psi, \\
\text { where } \varepsilon_{x x}^{0}=u^{\prime}, \chi=-\psi^{\prime} \text { and } \gamma=w^{\prime}-\psi
\end{gathered}
$$

where $\gamma$ is the transverse shear strain. $\psi$ denotes the independent rotation of the cross-section. The underlying assumptions are that plane cross-sections before deformation remain plane after deformation, but not necessarily perpendicular to the neutral beam axis, and that the shear angle is uniformly distributed over the depth of the cross-section. The Euler-Bernoulli model is found for inextensible shear strain by setting $\gamma=0$. The buckling of inextensible Timoshenko columns, obtained by setting $\varepsilon_{x x}^{0}=0$, can be studied by two different theories, the Engesser theory [38] and the Haringx theory [39], which differ concerning how the normal and shear forces are defined with respect to the non-deformed or deformed cross-section. In the Engesser theory, the normal force is chosen parallel to the beam axis in the loaded state, whereas in the Haringx approach, the normal force is chosen normal to the deformed cross-section in the state after loading. As shown by Bažant [40] (see also [41]; see more recently [42]), the differences between these shear theories can be understood as the difference of the strain measures definition. Bažant and Beghini $[43,44]$ discussed the validity of each shear beam-column theory. When a constant shear modulus $G$ is used, the correct small-strain critical load of a shear-deformable column is obtained only with the Engesser theory, the critical load of a soft in shear transversely layered column (such as the elastomeric or seismic isolation bearing) only with the Haringx theory, and the critical load of a biaxially compressed highly orthotropic or layered body soft in shear only with a mixture of both. The buckling of extensional Timoshenko columns has been studied by Ziegler [45] for an Engesser based model, later generalized by Reissner [46], for both Engesser and Haringx based models. The results of Reissner [46] are based on the general in-plane theory that Reissner derived in 1972 [47]. The post-buckling of extensional Timoshenko columns is studied by Goto et al. [48] using elliptic integrals, as a generalization of the Elastica problem of Euler.

The Timoshenko beam-column model requires shear correction factors to compensate for the error due to the constant shear stress assumption. A higher-order shear beam-column theory has been developed to refine the simplified kinematics of the Timoshenko model by expressing the relationships with polynomials of higher degree. In this refined theory, the transverse shear strain (and shear stress) is vanishing on the top and bottom edges of the beam.

$\varepsilon_{x x}=u^{\prime}-z \psi^{\prime}-\frac{4}{3 h^{2}} z^{3}\left(w^{\prime \prime}-\psi^{\prime}\right)=\varepsilon_{x x}^{0}+z \chi+z^{3} \rho \quad$ and

$2 \varepsilon_{x z}=\left(1-\frac{4 z^{2}}{h^{2}}\right)\left(w^{\prime}-\psi\right)$ where $\varepsilon_{x x}^{0}=u^{\prime}$,

$\chi=-\psi^{\prime}, \gamma=w^{\prime}-\psi$ and $\rho=-\frac{4}{3 h^{2}}\left(w^{\prime \prime}-\psi^{\prime}\right)$

where $h$ is the height of the cross-section and $\rho$ is a higherorder shear strain measure. This theory is classified as the Bickford-Reddy theory (see [49-52]). Some other refined shear deformation theories can be found in the literature, as detailed 
for instance by Ghugal and Shimpi [53]. A variationally consistent higher-order shear beam theory has been recently presented by Shi and Voyiadjis [54]. The buckling of inextensible Bickford-Reddy column is studied by Wang et al. [55].

\subsubsection{Partial composite beam-column theories}

The same hierarchical classification can be presented for the partial composite beam-column theories, i.e. for composite members connected with some shear interaction law. The simplest partial composite beam-column model is that based on the Euler-Bernoulli model for each sub-element with the following kinematics:

$\varepsilon_{x x, 1}=u_{1}^{\prime}-z_{1} w_{1}^{\prime \prime}=\varepsilon_{x x, 1}^{0}+z_{1} \chi_{1} \quad$ and

$\varepsilon_{x x, 2}=u_{2}^{\prime}-z_{2} w_{2}^{\prime \prime}=\varepsilon_{x x, 2}^{0}+z_{2} \chi_{2}$

where the individual coordinate axis is located at the centroid of each individual cross-section. This is a generalization of Eq. (11) for the two connected Euler-Bernoulli sub-beam-columns.

The curvatures of the two sub-elements are supposed to be equal, i.e. the following kinematical constraint is enforced:

$w_{1}^{\prime \prime}=w_{2}^{\prime \prime}=w^{\prime \prime}$.

The buckling solution of the partial composite Euler-Bernoulli column has been found by Hoff and Mautner [13] for the sandwich beam, or [3] for the partial composite beam for simple boundary conditions. General boundary conditions are treated by Girhammar and Pan [2]. The buckling solution of the Euler-Bernoulli column based on the Reissner model for each subelement of the composite column (with additional extensibility effect) has been recently considered by Kryžanowski et al. [56] and Schnabl and Planinc [7]. A more sophisticated partial composite beam-column model is that based on the Timoshenko shear model with the following kinematics:

$\varepsilon_{x x, 1}=u_{1}^{\prime}-z_{1} \psi_{1}^{\prime}=\varepsilon_{x x, 1}^{0}+z_{1} \chi_{1}, \quad 2 \varepsilon_{x z, 1}=w_{1}^{\prime}-\psi_{1} \quad$ and

$\varepsilon_{x x, 2}=u_{2}^{\prime}-z_{2} \psi_{2}^{\prime}=\varepsilon_{x x, 2}^{0}+z_{2} \chi_{2}, \quad 2 \varepsilon_{x z, 2}=w_{2}^{\prime}-\psi_{2}$.

Moreover, in the simplest model of partial composite Timoshenko columns, both the curvatures of and the shear deformations in the two connected sub-elements are assumed to be equal, i.e. the following kinematical constraints apply:

$w_{1}^{\prime \prime}=w_{2}^{\prime \prime}=w^{\prime \prime}$ and $\psi_{1}=\psi_{2}=\psi$.

The buckling of partial composite Timoshenko columns based on the Engesser theory is studied by $\mathrm{Xu}$ and $\mathrm{Wu}$ [6] with such a uniform shear model (equal shear deformations in the sub-elements). Considering a uniform shear model based on Eq. (17) has the advantage of reducing the degrees-of-freedom of the structural problem (an assumption strictly valid only for two identical sub-elements). In this paper we discuss the corresponding buckling theory, by using a variational approach. Also, our study will be also extended to include the partial composite Timoshenho model based on the Haringx theory with a different definition of the normal and shear forces. Furthermore, we will in the present paper develop new analytical buckling solutions when assuming different shear angles for the two subelements of the partial composite column, i.e. based on the following kinematical constraints:

$w_{1}^{\prime \prime}=w_{2}^{\prime \prime}=w^{\prime \prime}$ and $\psi_{1} \neq \psi_{2}$.

Such refined kinematics has already been considered for the case of beam bending by Schnabl et al. [57]. However, very recently, Schnabl and Planinc [8] obtained some buckling solutions with such a shear differential model (individual shear deformations in each sub-element) based on the Haringx theory. Finally, Krawczyk

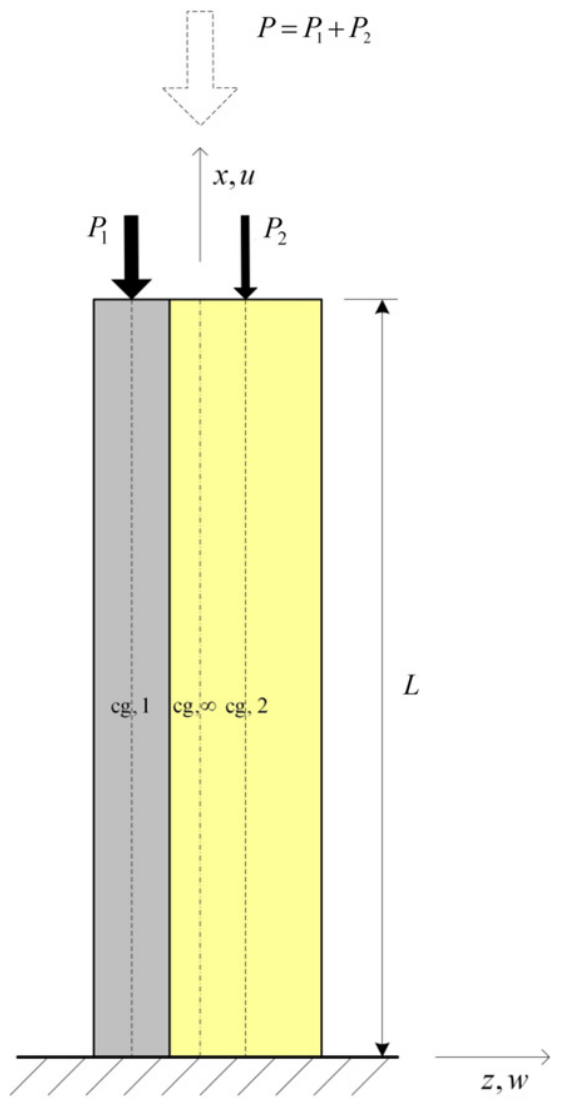

Fig. 3. A partial composite column with interlayer slip subjected to two axial loads applied at the centroid of each sub-element. No pre-buckling requires $P_{1}=$ $\left(E_{1} A_{1} / E A_{0}\right) P$ and $P_{2}=\left(E_{2} A_{2} / E A_{0}\right) P$ or that $P=P_{1}+P_{2}$ is applied at cg, $\infty$.

et al. [29] (see also [58]) investigated the higher-order shear model for partial composite beams, based on parabolic shear strain distribution over the cross-section.

The most general model for partial composite beam-columns based on the Bickford-Reddy theory can be written as:

$\varepsilon_{x x, 1}=u_{1}^{\prime}-z_{1} \psi_{1}^{\prime}-\frac{4}{3 h^{2}} z_{1}^{3}\left(w^{\prime \prime}-\psi_{1}^{\prime}\right)$

$2 \varepsilon_{x z, 1}=\left(1-\frac{4 z_{1}^{2}}{h^{2}}\right)\left(w^{\prime}-\psi_{1}\right)$ and

$\varepsilon_{x x, 2}=u_{2}^{\prime}-z_{2} \psi_{2}^{\prime}-\frac{4}{3 h^{2}} z_{2}^{3}\left(w^{\prime \prime}-\psi_{2}^{\prime}\right)$,

$2 \varepsilon_{x z, 2}=\left(1-\frac{4 z_{2}^{2}}{h^{2}}\right)\left(w^{\prime}-\psi_{2}\right)$.

\subsection{Mode of application of axial loadings}

The stability of partial composite Euler-Bernoulli columns composed of two connected sub-elements (Fig. 3) or a sandwich construction (Fig. 4) subjected to axial compressive loads is first investigated. In the general case, the composite beam-column is subjected to two independent load parameters $\left(P_{1}, P_{2}\right)$ acting along the centroidal axis of each sub-element. However, in order to induce no pre-bending of the beam-column, i.e. to induce no eccentricity moment at the point of load application, the relationship between $P_{1}$ and $P_{2}$ should be such to cause only uniform axial strain in the member and no bending deformation.

The sum of the two axial forces in the sub-elements is equal to the total axial load on the composite member:

$P_{1}+P_{2}=P$. 


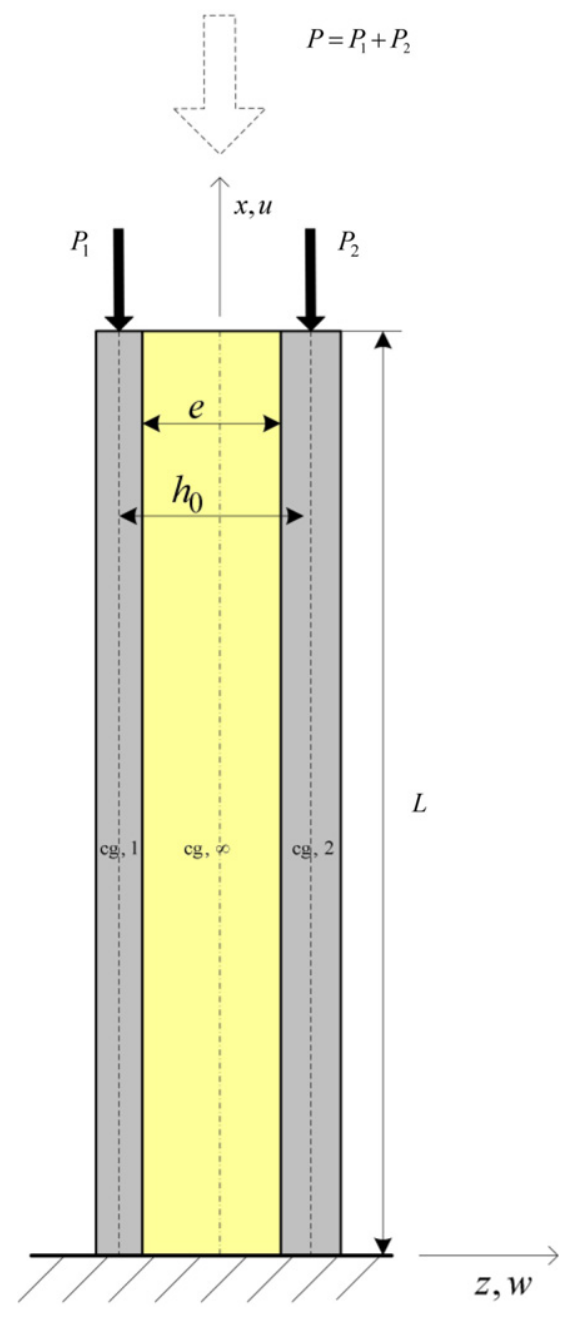

Fig. 4. A sandwich column with a core weak in shear subjected to two axial loads applied at the centroid of the faces.

Equal axial strain in the sub-elements requires that

$\varepsilon_{1}=-\frac{P_{1}}{E_{1} A_{1}}=-\frac{P_{2}}{E_{2} A_{2}}=\varepsilon_{2}$

i.e. the axial load on respective sub-element must equal

$P_{1}=\frac{E_{1} A_{1}}{E A_{0}} P$ and $\quad P_{2}=\frac{E_{2} A_{2}}{E A_{0}} P$

in order to ensure that no eccentricity moment arises or that no pre-bending occurs, i.e. $w_{1}=w_{2}=w=0$. This loading arrangement is equivalent to the total axial load $P$ acting in the centroid of the full composite section (cf. [1]).

The same conditions and conclusions are valid also for the buckling problem of the sandwich beam-column (Fig. 4).

\subsection{Aim and scope}

In this paper, variational formulations of the stability of partial composite columns (or sandwich columns) under different conditions are presented in order to derive the governing differential equations and at the same time obtain the pertaining relevant boundary conditions. We show the equivalence between the Hoff sandwich theory, the partial composite theory with interlayer slip and the Bickford-Reddy theory, from a stability point of view. Different modes of application of the axial loads and their effects are investigated. The results are compared to those of Sokolinsky and Frostig [59], who also show the influence of the specific mode of axial loading on the buckling of sandwich panels. Some specific effects, as axial extensibility and shear contribution are analysed and compared with the classical theory of composite beam-columns with partial interaction.

\section{General theory for partial composite Euler-Bernoulli beam- columns-a variational solution for the reference case}

In this section, we will derive the basic equations for partial composite beam-columns using the Euler-Bernoulli theory for each sub-element. This case will be used as a reference case to compare with the other theories developed in the paper. These equations have earlier been presented in the literature, see e.g. [5,2], but they were not derived using the variational approach in the same way as presented in the present paper. These fundamental equations are often presented as a sixth-order differential equation with the deflection $w(x)$ as the variable. In this paper we will also present for the first time the corresponding fourth-order differential equations including second-order effects with the internal normal forces $N_{1}(x)$ and $N_{2}(x)$ as the variables (the corresponding equations were derived in [1], but they were not explicitly formulated as presented in this paper).

As mentioned before, the differential equations derived below are also valid for sandwich beams, usually composed of three layers, two thin faces and a thick weak shear core [9-11]. The static in-plane behaviour of sandwich beams is well established by the work of Hoff [12].

A free-body diagram of an element in the composite beamcolumn is shown in Fig. 5 , where moments $\left(M, M_{1}, M_{2}\right)$, shear forces $\left(V, V_{1}, V_{2}\right)$, normal forces $\left(N_{1}, N_{2}\right)$, slip force per unit length $\left(V_{s}=K \cdot \Delta u\right)$ are defined. The influence of shear forces will be neglected in the first part of the study. We consider the beam-column loaded by axial forces of magnitude $P_{1}$ and $P_{2}$ applied at the centroid of sub-element 1 and 2 , respectively $\left(P_{1}+\right.$ $P_{2}=P$ ) and a distributed transverse load $q(x)$ (a generalization of the structural problem illustrated in Figs. 3 and 4 ). The general form of the equilibrium equations in the buckled state may be obtained from the linearized energy functional expressed as:

$$
\begin{aligned}
& U\left[u_{1}, u_{2}, w\right] \\
& =\int_{0}^{L}\left(\frac{1}{2} E_{1} I_{1} w^{\prime \prime 2}+\frac{1}{2} \overline{N_{1}} w^{\prime 2}+\overline{N_{1}} u_{1}^{\prime}+\frac{1}{2} E_{2} I_{2} w^{\prime \prime 2}\right. \\
& \left.\quad+\frac{1}{2} \overline{N_{2}} w^{\prime 2}+\overline{N_{2}} u_{2}^{\prime}+\frac{1}{2} K \cdot \Delta u^{2}\right) \mathrm{d} x \\
& \quad+P_{1} u_{1}(L)+P_{2} u_{2}(L)-\int_{0}^{L} q w \mathrm{~d} x
\end{aligned}
$$

where $\overline{N_{i}}$ are the linearized normal force in the $i$ th sub-element. Note that this energy functional is derived assuming an axially immovable support at the clamped end $x=0$, i.e. $u_{1}(0)=$ $u_{2}(0)=0$. These boundary conditions, specifically valid for an immovable clamped end, are not equivalent to the general boundary conditions used, for example, in [2], where the work of the applied axial forces (balanced forces at both ends) is included in the energy functional through the total shortening of the beam-column axis expressed in terms of the deflection $w$ (and not in terms of the axial displacements of the sub-elements $u_{i}$ ). The slip $\Delta u$ in the shear layer is related to the other kinematical parameters via (cf. [5]):

$\Delta u=u_{2}-u_{1}+h_{0} w^{\prime}$. 


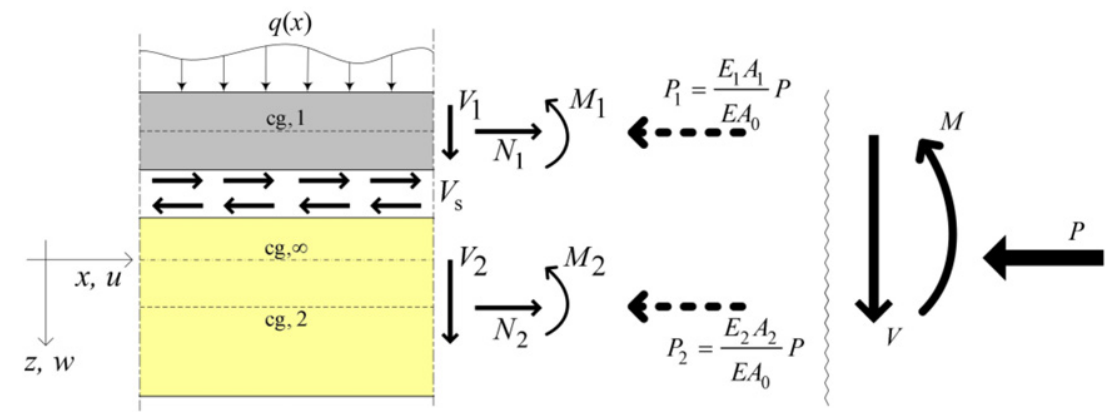

Fig. 5. Decomposition of the internal forces and moments in the partial composite beam-column (non-deformed state).

The linear elastic constitutive law for each sub-element and the linear elastic shear connection law are written as:

$$
\left(\begin{array}{l}
N_{1} \\
M_{1} \\
N_{2} \\
M_{2} \\
V_{s}
\end{array}\right)=\left(\begin{array}{llcll}
E_{1} A_{1} & 0 & 0 & 0 & 0 \\
0 & E_{1} I_{1} & 0 & 0 & 0 \\
0 & 0 & E_{2} A_{2} & 0 & 0 \\
0 & 0 & 0 & E_{2} I_{2} & 0 \\
0 & 0 & 0 & 0 & K
\end{array}\right)\left(\begin{array}{l}
\varepsilon_{1} \\
\chi_{1} \\
\varepsilon_{2} \\
\chi_{2} \\
\Delta u
\end{array}\right)
$$

where $N_{i}$ are the internal normal forces and $M_{i}$ are the internal bending moment in the $i$ th sub-element, $V_{s}$ is the linearized slip force in the shear layer. The strain measures are given for this Euler-Bernoulli problem as:

$$
\left(\begin{array}{l}
\varepsilon_{1} \\
\chi_{1} \\
\varepsilon_{2} \\
\chi_{2} \\
\Delta u
\end{array}\right)=\left(\begin{array}{c}
u_{1}^{\prime} \\
-w_{1}^{\prime \prime} \\
u_{2}^{\prime} \\
-w_{2}^{\prime \prime} \\
u_{2}-u_{1}+h_{0} w^{\prime}
\end{array}\right)
$$

The variation of the total potential energy is written as:

$$
\begin{aligned}
\delta U= & \int_{0}^{L}\left[E I_{0} w^{\prime \prime} \delta w^{\prime \prime}+\overline{N_{1}}\left(\delta u_{1}^{\prime}+w^{\prime} \delta w^{\prime}\right)\right. \\
& \left.+\overline{N_{2}}\left(\delta u_{2}^{\prime}+w^{\prime} \delta w^{\prime}\right)+K \cdot \Delta u \cdot \delta \Delta u\right] \mathrm{d} x \\
& +P_{1} \delta u_{1}(L)+P_{2} \delta u_{2}(L)-\int_{0}^{L} q \delta w \mathrm{~d} x=0 .
\end{aligned}
$$

An integration by part leads to the following system of differential equations:

$$
\left\{\begin{array}{l}
E I_{0} w^{(4)}-\left[\left(\overline{N_{1}}+\overline{N_{2}}\right) w^{\prime}\right]^{\prime}-h_{0}\left(V_{s}\right)^{\prime}-q=0 \\
-\left(\overline{N_{1}}\right)^{\prime}-V_{s}=0 \\
-\left(\overline{N_{2}}\right)^{\prime}+V_{s}=0
\end{array}\right.
$$

with the following natural and essential boundary conditions:

$$
\begin{aligned}
& {\left[\left[-E I_{0} w^{\prime \prime \prime}+\left(\overline{N_{1}}+\overline{N_{2}}\right) w^{\prime}+h_{0} V_{s}\right] \delta w\right]_{0}^{L}=0} \\
& {\left[E I_{0} w^{\prime \prime} \delta w^{\prime}\right]_{0}^{L}=0} \\
& {\left[\overline{N_{1}} \delta u_{1}\right]_{0}^{L}+P_{1} \delta u_{1}(L)=0} \\
& {\left[\overline{N_{2}} \delta u_{2}\right]_{0}^{L}+P_{2} \delta u_{2}(L)=0 .}
\end{aligned}
$$

The second and the third differential equations of Eq. (28) lead to the simplification:

$$
\begin{aligned}
& \left(\overline{N_{1}}+\overline{N_{2}}\right)^{\prime}=0 \\
& \Rightarrow \overline{N_{1}}+\overline{N_{2}}=\overline{N_{1}}(L)+\overline{N_{2}}(L)=-P_{1}-P_{2}=-P .
\end{aligned}
$$

From Eq. (28), the differential equation for the deflection is finally obtained as

$$
w^{(6)}+\left(\frac{P}{E I_{0}}-\alpha^{2}\right) w^{(4)}-\alpha^{2} \frac{P}{E I_{\infty}} w^{\prime \prime}+\frac{\alpha^{2}}{E I_{\infty}} q-\frac{q^{\prime \prime}}{E I_{0}}=0 .
$$

Eq. (31) is exactly the equation obtained by Girhammar and Gopu [1] and Girhammar and Pan [2] in alternative ways (the sixth-order differential equation (31) was obtained without the use of additional Lagrange multipliers, a notable difference with the reasoning of Girhammar and Pan [2]). The buckling solutions are detailed in these two mentioned papers for various boundary conditions. Note that this differential equation may lead to some boundary layer phenomena, as recently investigated by Challamel and Girhammar [60].

For a pure column without transversal load $(q=0)$, the differential equation (31) is reduced to

$w^{(6)}+\left(\frac{P}{E I_{0}}-\alpha^{2}\right) w^{(4)}-\alpha^{2} \frac{P}{E I_{\infty}} w^{\prime \prime}=0$.

The buckling solution has been found by Hoff and Mautner [13] for the sandwich column, and by Möhler [3] for the partial composite column for simply supported boundary conditions. Independently and for general boundary conditions the buckling loads were found by Girhammar and Gopu [5] and Girhammar and Pan [2].

It is also possible to derive a corresponding differential equation expressed in terms of the internal normal forces $\overline{N_{1}}$ and $\overline{N_{2}}$, respectively, as

$$
\begin{aligned}
& {\overline{N_{1}}}^{(4)}+\left(\frac{P}{E I_{0}}-\alpha^{2}\right){\overline{N_{1}}}^{\prime \prime}-\alpha^{2} \frac{P}{E I_{\infty}} \overline{N_{1}}-\beta \frac{P^{2}}{E_{2} A_{2} h_{0}}+\beta q \\
& =0 \\
& {\overline{N_{2}}}^{(4)}+\left(\frac{P}{E I_{0}}-\alpha^{2}\right){\overline{N_{2}}}^{\prime \prime}-\alpha^{2} \frac{P}{E I_{\infty}} \overline{N_{2}}-\beta \frac{P^{2}}{E_{1} A_{1} h_{0}}-\beta q \\
& =0 .
\end{aligned}
$$

It is easy to check that the fundamental homogeneous solution with the constant $\overline{N_{1}}(x)=-P\left(E_{1} A_{1} / E A_{0}\right)=-P_{1}$ and $\overline{N_{2}}(x)=$ $-P\left(E_{2} A_{2} / E A_{0}\right)=-P_{2}$ verifies Eqs. (33) and (34), respectively, in the case without lateral loading $(q=0)$. This fundamental solution is also compatible with the boundary conditions $\overline{N_{1}}(L)=$ $-P_{1}$ and $\overline{N_{2}}(L)=-P_{2}$, respectively. Note that this is the unique homogeneous solution for this problem with the loading system as presented in Figs. 3 and 4.

\section{A remark on the partial composite or sandwich column theory and the Bickford-Reddy column theory}

We shall now discuss a peculiar mathematical similarity between the Euler-Bernoulli model for partial composite or sandwich columns and the Bickford-Reddy model for ordinary solid columns. The in-plane buckling problem of the partial composite column (or sandwich column) is governed by a sixthorder differential equation given by Eq. (32). For pinned end 
columns, clamped-free columns or clamped-clamped columns, the buckling load is given by [2]:

$\frac{P_{\mathrm{cr}}}{P_{E}^{0}}=\frac{P_{E}^{0}+\alpha^{2} E I_{0}}{P_{E}^{0}+\frac{E I_{0}}{E I_{\infty}} \alpha^{2} E I_{0}}=\frac{E I_{\infty}}{E I_{0}} \frac{1+\frac{P_{E}^{0}}{\alpha^{2} E I_{0}}}{1+\frac{E I_{\infty}}{E I_{0}} \frac{P_{E}^{0}}{\alpha^{2} E I_{0}}}$

where $P_{E}^{0}$ is the Euler buckling load for the non-composite section. It is worth mentioning that such a formulae as Eq. (35) cannot be written directly in the format of the Engesser or the Haringx formula $[38,39]$. The reader is referred to the discussion of Bažant [61], for the analysis of the relevancy of each formulae in case of sandwich column buckling. As shown by Blaauwendraad [19], for instance, for sandwich beam-columns, Eq. (35) can be reformulated as:

$\frac{P_{\mathrm{cr}}}{P_{E}^{0}}=1+\frac{\frac{1}{P_{E}^{0}}}{\frac{1}{P_{0}}+\frac{1}{P_{S}}} \quad$ with $\frac{P_{0}}{P_{E}^{0}}=\frac{E I_{\infty}}{E I_{0}}-1$ and

$P_{S}=E I_{0} \alpha^{2}\left(1-\frac{E I_{0}}{E I_{\infty}}\right)$.

Eq. (36) is sometimes refereed to as a generalized Engesser based formulae. In the case of the clamped-free column considered in the paper, this load is simply given by:

$P_{E}^{0}=E I_{0}\left(\frac{\pi}{2 L}\right)^{2}$.

Note that Eq. (35) is not applicable in case of clamped-pinned columns, for instance, as detailed by Girhammar and Pan [2] from their results obtained for partial composite Euler-Bernoulli columns with the four Euler boundary conditions. This phenomenon is analogous to the one observed by Plantema [10] and Ziegler [45] for buckling of clamped-pinned Timoshenko columns (their studies revealed the particular characteristics of clamped-pinned boundary conditions).

It is easy to check for instance the following asymptotic properties:

$\alpha=0 \Rightarrow \frac{P_{\mathrm{cr}}}{P_{E}^{0}}=1 \quad$ and $\quad \alpha \rightarrow \infty \Rightarrow \frac{P_{\mathrm{cr}}}{P_{E}^{0}} \rightarrow \frac{E I_{\infty}}{E I_{0}}$.

Eqs. (35) and (36) can also be presented in the following format:

$\frac{P_{\mathrm{cr}}}{P_{E}^{\infty}}=\frac{1+\frac{P_{E}^{\infty}}{\alpha^{2} E I_{\infty}}}{1+\frac{P_{E}^{\infty}}{\alpha^{2} E I_{0}}} \quad$ with $P_{E}^{\infty}=E I_{\infty}\left(\frac{\pi}{2 L}\right)^{2}$

where $P_{E}^{\infty}$ is the Euler buckling load for the full composite section. The sixth-order differential equation obtained in the Euler-Bernoulli based composite theory with partial interaction (or sandwich beam theory of Hoff) is close to the sixth-order differential equation obtained for the solid beam Bickford-Reddy theory $[50,55,62]$. It can be shown that the sixth-order differential equations of both theories are strictly equivalent if some specific changes of parameters are made. In fact, Wang et al. [55] (or Wang et al. [62]) show that for pinned end columns, fixed end columns and symmetrically elastic rotationally restrained end columns, the Bickford-Reddy critical load is related to the Euler critical load by:

$\frac{P_{\mathrm{cr}}}{P_{E}}=\frac{1+\frac{P_{E}}{P^{*}}}{1+\frac{P_{E}}{P^{* *}}} \quad$ with

$P^{*}=G \bar{A} \frac{D_{x x}^{2}}{\alpha_{R}^{2} \tilde{D}_{x x}}$ and $P^{* *}=G \bar{A} \frac{D_{x x}}{\bar{D}_{x x}}$

where the notations of Wang et al. [55] have been used. $P_{E}$ is the Euler-Bernoulli buckling load. $G$ is the shear modulus, $\bar{A}=$
$A_{R}-2 \beta_{R} I_{R}+\beta_{R}^{2} J_{R}, D_{x x}=E I_{R}, \bar{D}_{x x}=E\left(I_{R}-2 \alpha_{R} J_{R}+\alpha_{R}^{2} K_{R}\right)$, and $\tilde{D}_{x x}=E^{2}\left(K_{R} I_{R}-J_{R}^{2}\right)$, where $\alpha_{R}=4 /\left(3 h^{2}\right), \beta_{R}=4 / h^{2}$, and $\left(A_{R}, I_{R}, J_{R}, K_{R}\right)=\int_{A}\left(1, z^{2}, z^{4}, z^{6}\right) \mathrm{d} A$.

Clearly, both theories are equivalent if the following parameters are interchanged in Eqs. (39) and (40):

$P^{*}=\alpha^{2} E I_{\infty}, \quad P^{* *}=\alpha^{2} E I_{0} \quad$ and

$P_{E}=P_{E}^{\infty}=E I_{\infty}\left(\frac{\pi}{2 L}\right)^{2}$.

Then, the following parameters can be identified:

$E I_{\infty}=D_{x x}, \quad E I_{0}=D_{x x} \frac{P^{* *}}{P^{*}}=\frac{\alpha_{R}^{2} \tilde{D}_{x x}}{\bar{D}_{x x}}$ and

$\alpha^{2}=\frac{P^{*}}{D_{x x}}=G \bar{A} \frac{D_{x x}}{\alpha_{R}^{2} \tilde{D}_{x x}}$.

Therefore, there is an equivalence between the partial composite Euler-Bernoulli beam-column theory (or the Hoff theory) and Bickford-Reddy higher-order beam-column theory. With this correspondence, the sixth-order differential equation of Bickford-Reddy theory (see [55]) can be written in the same format as Eq. (32):

$w^{(6)}+\left(\frac{\bar{D}_{x x} P}{\alpha_{R}^{2} \tilde{D}_{x x}}-G \bar{A} \frac{D_{x x}}{\alpha_{R}^{2} \tilde{D}_{x x}}\right) w^{(4)}-G \bar{A} \frac{P}{\alpha_{R}^{2} \tilde{D}_{x x}} w^{\prime \prime}=0$.

However, we have to mention that the boundary conditions for the two theories are not strictly equivalent. Furthermore, it is noted that the kinematics of the two beam-column theories are firmly different, as is evident from the presentation given in Section 1 of this paper. One theory leading to a piecewise linear strain distribution over the cross-section of each subelement (partial composite Euler-Bernoulli beam-column theory) whereas the second one is associated with a nonlinear shear strain distribution (ordinary solid Bickford-Reddy beam-column theory). The analogy between the two theories is mathematically of a formal nature.

\section{Buckling of partial composite columns $(q=0)$}

In the case of a clamped-free column, the boundary conditions derived from Eq. (29) are:

$w(0)=0$ and $\left[E I_{0} w^{\prime \prime \prime}+P w^{\prime}-h_{0} V_{s}\right](L)=0$

$w^{\prime}(0)=0$ and $w^{\prime \prime}(L)=0$

$u_{1}(0)=0$ and $\overline{N_{1}}(L)=-P_{1}$

$u_{2}(0)=0$ and $\overline{N_{2}}(L)=-P_{2}$.

Integration of the differential equation (28) together with Eq. (30) and the boundary condition according to the second condition of Eq. (44a) leads to the following differential equation (cf. this equation with the boundary expression given in Eq. (29a)):

$E I_{0} w^{\prime \prime \prime}+P w^{\prime}-h_{0} V_{s}=0$.

Therefore, the boundary condition at the clamped section $(x=$ 0 ) can be presented in the following simplified form by using Eqs. (44b)-(44d) and (45):

$u_{1}(0)=u_{2}(0)=w^{\prime}(0)=0 \Rightarrow V_{s}(0)=0 \Rightarrow w^{\prime \prime \prime}(0)=0$.

The boundary condition associated with the normal forces can be expressed in terms of the deflection in the following way by using Eqs. (28) (for $q=0$ ):

$E I_{0} w^{(4)}+\left(P-K h_{0}^{2}\right) w^{\prime \prime}-h_{0} K\left(\frac{\overline{N_{2}}}{E_{2} A_{2}}-\frac{\overline{N_{1}}}{E_{1} A_{1}}\right)=0$. 
Therefore, the boundary condition at $x=L$ dealing with the normal forces is written as:

$E I_{0} w^{(4)}(L)+h_{0} K\left(\frac{P_{2}}{E_{2} A_{2}}-\frac{P_{1}}{E_{1} A_{1}}\right)=0$.

The buckling problem without pre-bending deflection is based on the decomposition of the total load given by Eq. (22), and, thus, Eq. (48) can be reduced to:

$E I_{0} w^{(4)}(L)=0$.

Integrating Eq. (32), differentiating Eq. (47), using Eq. (28) together with the boundary conditions at the clamped end $(x=0)$ according to Eq. (47) (giving $w^{(5)}(0)=0$ ), the first condition of Eq. (44a) and the repeated use of these equations and results, the following fifth-order differential equation is obtained

$w^{(5)}+\left(\frac{P}{E I_{0}}-\alpha^{2}\right) w^{\prime \prime \prime}-\alpha^{2} \frac{P}{E I_{\infty}} w^{\prime}=0$.

This fifth-order differential equation is similar to the one introduced by Girhammar and Gopu [4], or more recently by Kryžanowski et al. [56] (see also [7]). The five boundary conditions can be summarized as:

$w(0)=w^{\prime}(0)=w^{\prime \prime \prime}(0)=w^{\prime \prime}(L)=0$ and $\quad w^{(4)}(L)=0$.

The general solution of Eq. (50) can be written as (see for instance [2]):

$$
\begin{aligned}
w(x)= & C_{1} \sinh \left(\theta_{1} x\right)+C_{2} \cosh \left(\theta_{1} x\right) \\
& +C_{3} \sin \left(\theta_{2} x\right)+C_{4} \cos \left(\theta_{2} x\right)+C_{5}
\end{aligned}
$$

where

$$
\left\{\begin{array}{l}
\theta_{1}=\sqrt{\frac{1}{2}\left\{\left(\alpha^{2}-\frac{P}{E I_{0}}\right)+\sqrt{\left.\left(\alpha^{2}-\frac{P}{E I_{0}}\right)^{2}+4 \alpha^{2} \frac{P}{E I_{\infty}}\right\}}\right.} \\
\theta_{2}=\sqrt{-\frac{1}{2}\left\{\left(\alpha^{2}-\frac{P}{E I_{0}}\right)-\sqrt{\left.\left(\alpha^{2}-\frac{P}{E I_{0}}\right)^{2}+4 \alpha^{2} \frac{P}{E I_{\infty}}\right\}}\right.} .
\end{array}\right.
$$

The five boundary conditions (51) finally leads to:

$C_{1}=C_{2}=C_{3}=0, \quad C_{5}=-C_{4} \quad$ and $\quad \theta_{2}=\frac{\pi}{2 L}$.

The buckling mode is finally written as:

$w(x)=C_{4}\left[\cos \left(\theta_{2} x\right)-1\right]$.

This buckling mode is similar to that of a homogeneous clampedfree Euler-Bernoulli column. It is easy to check that Eq. (35) is found again from the buckling load given by Eq. (53), which can be presented as (see Fig. 6):

$$
\frac{P_{\mathrm{cr}}}{P_{E}^{0}}=\frac{\left(\frac{\pi}{2}\right)^{2}+(\alpha L)^{2}}{\left(\frac{\pi}{2}\right)^{2}+\frac{E I_{0}}{E I_{\infty}}(\alpha L)^{2}} .
$$

The buckling load is a monotonically increasing function versus the dimensionless shear connection parameter denoted by $\alpha L$ according to Fig. 6. The buckling load tends towards that of the full composite column for infinite shear connector stiffness $(\alpha L(K) \rightarrow \infty)$.

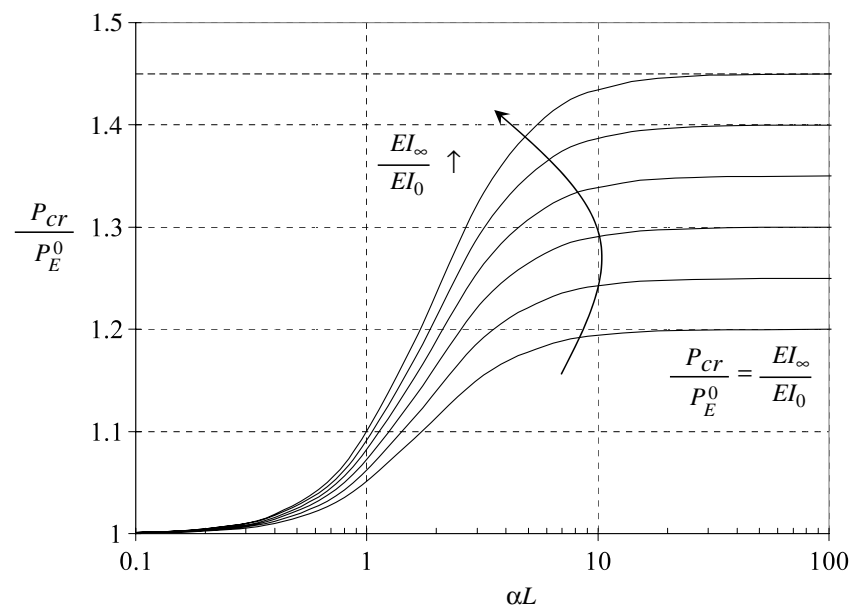

Fig. 6. Relative buckling load for partial composite Euler-Bernoulli columns versus the shear connection parameter $\alpha L$. Curves shown for different relative bending stiffness values $E I_{\infty} / E I_{0} \in\{1.2 ; 1.25 ; 1.3 ; 1.35 ; 1.4 ; 1.45 ; 1.5\}$.

\section{Application of eccentric axial loadings}

In practical applications, it is not uncommon that the axial forces are introduced in the composite column in different ways. For example, there are situations when the total axial load is introduced in only one sub-element or when the individual axial loads are applied arbitrarily on the individual sub-elements. Therefore, in this section we study some loading cases when different types of eccentricities at the top of the partial composite column are involved due to the axial loading. Eccentric axial loadings are referred to load configurations when the total axial load on the partial composite column is applied outside the centroid of the full composite member or when the individual axial loads are applied on the individual sub-elements outside their individual centroids.

5.1. Opposing eccentric axial loads applied on the individual subelements

Consider the load configuration in Fig. 7. The individual axial loads $\left(P_{1}\right.$ and $\left.P_{2}\right)$ are applied outside on the opposing sides of the centroid of each sub-element. We will show that this loading case is identical with respect to the buckling load with the loading case when a composite column is subjected to the total axial load $\left(P_{1}+P_{2}=P\right)$ applied at the centroid of the full composite section if the induced opposing eccentricity moments are balanced.

For this case, Eq. (27) is modified with regard to the external work as:

$$
\begin{aligned}
\delta U= & \int_{0}^{L}\left[E I_{0} w^{\prime \prime} \delta w^{\prime \prime}+\overline{N_{1}}\left(\delta u_{1}^{\prime}+w^{\prime} \delta w^{\prime}\right)\right. \\
& \left.+\overline{N_{2}}\left(\delta u_{2}^{\prime}+w^{\prime} \delta w^{\prime}\right)+K \cdot \Delta u \delta \Delta u\right] \mathrm{d} x \\
& +P_{1} \delta u_{1}(L)+P_{2} \delta u_{2}(L) \\
& +P_{1} e_{1} \delta w^{\prime}(L)-P_{2} e_{2} \delta w^{\prime}(L)-\int_{0}^{L} q \delta w \mathrm{~d} x=0 .
\end{aligned}
$$

The variational equation (56) is exactly equal to Eq. (27) if the eccentricities are related by the following mathematical relationship:

$\frac{e_{1}}{e_{2}}=\frac{P_{2}}{P_{1}}=\frac{E_{2} A_{2}}{E_{1} A_{1}}$.

This relationship ensures that no overall bending moment occurs in the composite beam-column. Therefore, the previous buckling 


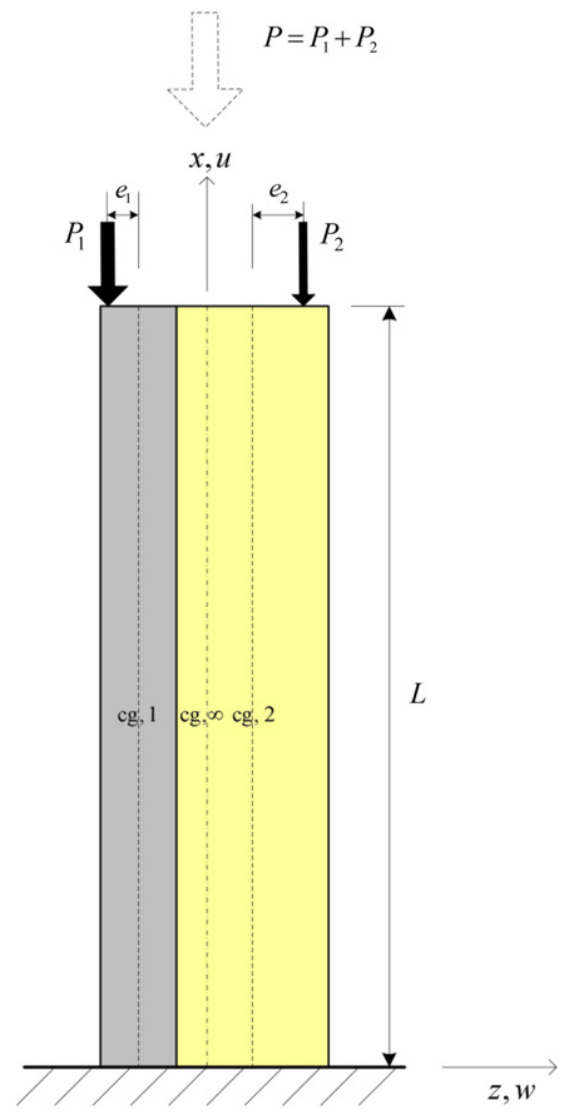

Fig. 7. A partial composite beam-column with interlayer slip subjected to two axial loads applied at opposing sides of the centroid of the sub-elements inducing opposing eccentricity moments at the top.

load Eq. (35) are still valid, i.e. introducing these eccentricities does not change the structural buckling problem, and the fundamental solution does not present any overall pre-bending deformations. Note, however, that in a real situation these eccentricity moments at the boundary need to be included in the local design at the top end of the composite member, even though they do not affect the overall buckling load of the system.

\subsection{Total axial load applied on a single sub-element}

Consider now the loading case according to Fig. 8. The total axial load $(P)$ on the partial composite column is applied on the centroid of only one of the sub-components $\left(P_{1}=P\right)$ (this loading configuration was studied by Girhammar and Gopu [4]). This case is characterized by a pre-bending state before buckling even for infinitely small magnitudes of the axial loading $P_{1}=P$. In fact, the tangential load component on the top surface of the composite column is no more vanishing for this load configuration. For this case, the variational principle is expressed as:

$$
\begin{aligned}
\delta U= & \int_{0}^{L}\left[E I_{0} w^{\prime \prime} \delta w^{\prime \prime}+\overline{N_{1}}\left(\delta u_{1}^{\prime}+w^{\prime} \delta w^{\prime}\right)\right. \\
& \left.+\overline{N_{2}}\left(\delta u_{2}^{\prime}+w^{\prime} \delta w^{\prime}\right)+K \cdot \Delta u \delta \Delta u\right] \mathrm{d} x \\
& +P \delta u_{1}(L)-\int_{0}^{L} q \delta w \mathrm{~d} x=0 .
\end{aligned}
$$

Previous differential equation (50) is still valid, but now the boundary conditions (51) are different. Referring to Eq. (48) and using the boundary condition $P_{2}=0$, the new boundary conditions

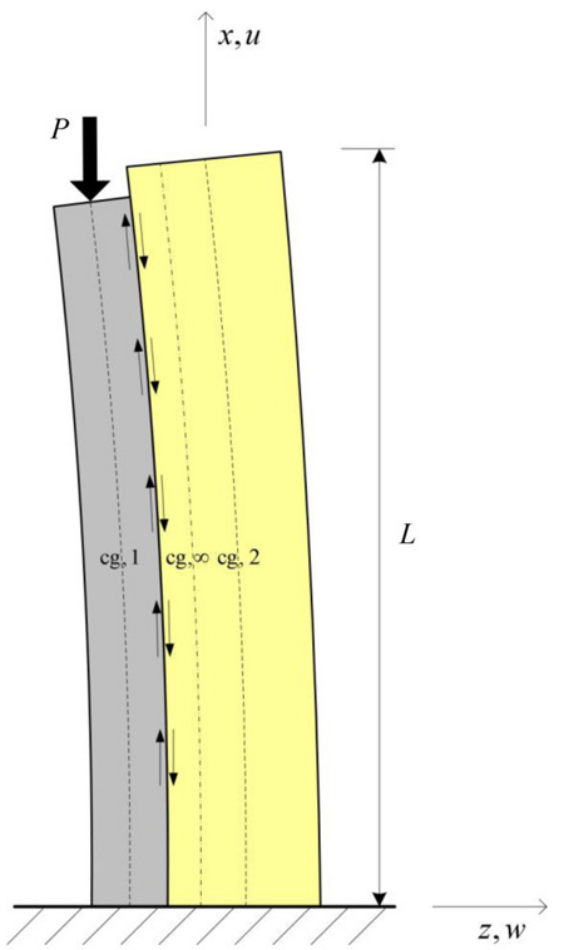

Fig. 8. A partial composite beam-column subjected to an axial load applied only at the centroid of sub-element $1, P_{1}=P$ and $P_{2}=0$. This load application introduces an eccentricity end moment at the top, $M_{\text {end }}=P \times z_{\mathrm{cg}, \infty}$.

can be written as:

$w(0)=w^{\prime}(0)=w^{\prime \prime \prime}(0)=w^{\prime \prime}(L)=0$ and

$w^{(4)}(L)-\beta \frac{P}{E_{1} A_{1}}=0$.

The boundary conditions at the clamped section $(x=0)$ lead to a simplification of the problem:

$w^{\prime}(0)=w^{\prime \prime \prime}(0)=0 \Rightarrow C_{1}=C_{3}=0, \quad$ which together with

$w(0)=0 \Rightarrow C_{5}=-C_{2}-C_{4}$.

Therefore, the deflection in this case is expressed as:

$w(x)=C_{2}\left[\cosh \left(\theta_{1} x\right)-1\right]+C_{4}\left[\cos \left(\theta_{2} x\right)-1\right]$.

Note that the deflection mode is affected by the mode of loading as the parameter $C_{2}$ in this case is not vanishing. The two last boundary conditions in Eq. (59) lead to the linear equation system:

$$
\left\{\begin{array}{l}
C_{2} \theta_{1}^{2} \cosh \left(\theta_{1} L\right)-C_{4} \theta_{2}^{2} \cos \left(\theta_{2} L\right)=0 \\
C_{2} \theta_{1}^{4} \cosh \left(\theta_{1} L\right)+C_{4} \theta_{2}^{4} \cos \left(\theta_{2} L\right)=\beta \frac{P}{E_{1} A_{1}} .
\end{array}\right.
$$

Note that this equation system is singular for the buckling load of the "perfect loading system", namely $\theta_{2, \mathrm{cr}}=\pi / 2 L$. The buckling load is identical to the previous case, but there is no fundamental path without deflection before buckling occurs. The beam-column behaves like an imperfect ordinary beam-column (see [63]), where the deflection tends towards an infinite value, as the load tends towards the buckling load, but there is deflection during the whole process before buckling. This theoretical analysis is consistent with the recent numerical analysis of Ranzi et al. [64], who also studied a similar case of a single axial loading on one of the sub-components. 


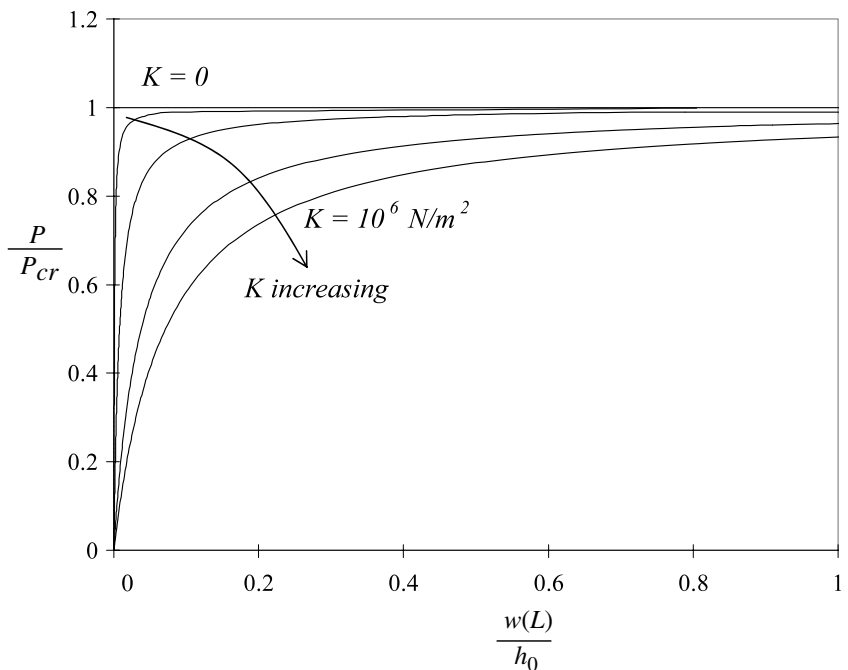

Fig. 9. Relative load-deflection relationships versus the slip modulus $K \in\left\{0 ; 10^{4}\right.$; $\left.10^{5} ; 5 \times 10^{5} ; 10^{6}\right\} \mathrm{N} / \mathrm{m}^{2}$.

Resolution of this linear system gives the load-dependent coefficients of the deflection:

$$
\left\{\begin{array}{l}
C_{2}=\beta \frac{P}{E_{1} A_{1}} \frac{1}{\theta_{1}^{2}\left(\theta_{1}^{2}+\theta_{2}^{2}\right)} \frac{1}{\cosh \left(\theta_{1} L\right)} \\
C_{4}=\beta \frac{P}{E_{1} A_{1}} \frac{1}{\theta_{2}^{2}\left(\theta_{1}^{2}+\theta_{2}^{2}\right)} \frac{1}{\cos \left(\theta_{2} L\right)} .
\end{array}\right.
$$

Finally, the load-deflection response is given for this problem under the second-order assumption as:

$$
\frac{w(L)}{h_{0}}=\frac{\beta}{h_{0}} \frac{P}{E_{1} A_{1}} \frac{1}{\theta_{1}^{2}+\theta_{2}^{2}}\left[\frac{\cosh \left(\theta_{1} L\right)-1}{\theta_{1}^{2} \cosh \left(\theta_{1} L\right)}+\frac{\cos \left(\theta_{2} L\right)-1}{\theta_{2}^{2} \cos \left(\theta_{2} L\right)}\right] .
$$

The curve corresponding to this load-displacement relationship is plotted in Fig. 9 (the negative deflection is counted positive in this figure). The mechanical and geometrical properties of the two-layer composite column are characterized by the following parameters: (1) elastic moduli of layers $E_{1}=E_{2}=8000 \mathrm{MPa}$; (2) interlayer-slip modulus $K \in\left\{0 ; 10^{4} ; 10^{5} ; 5 \times 10^{5} ; 10^{6}\right\} \mathrm{N} / \mathrm{m}^{2}$; (3) length of the column $L=5 \mathrm{~m}$; (4) layer heights $h_{1}=h_{2}=$ $0.1 \mathrm{~m}$; and (5) layer widths $b_{1}=b_{2}=0.2 \mathrm{~m}$. These parameters are identical to the ones used by Kryžanowski et al. [56] in their numerical study. The load parameter has been presented in a dimensionless form, although the buckling load depends on the interlayer-slip modulus according to Eq. (35).

The non-composite column $(K=0)$ behaves like the "perfect loading" case associated with the loading mode described by Fig. 3 or Fig. 4. Hence, the non-composite column is not sensitive to the loading mode, a conclusion also found by Girhammar and Gopu [4,1]. However, the mode of loading strongly influences the deflection response for partial and full composite columns (see Fig. 9). The influence on the deflection is more pronounced for higher values of the slip modulus $K$. The effects of the loading mode are also discussed by Sokolinsky and Frostig [59] for sandwich beam-columns. Sokolinsky and Frostig [59] also show the extreme sensitivity of the axial loading mode on the buckling behaviour. However, the fundamental difference between the present results, and the ones of Sokolinsky and Frostig [59], is that only the load-deflection curve is affected by the axial loading mode in the present paper. The buckling load is not affected by the change of loading mode although the load-deflection curve is affected.

As a conclusion, a column subjected to an axial load applied only on one of the sub-elements, behaves like a perfect column with respect to the buckling load, but like an imperfect column with respect to the pre-bending deformations before buckling due to the eccentricity moment at the top of the partial composite beam-column. The deflections tend towards an infinite value when the load tends towards the buckling load of the "perfect loading" case, which is an upper limit condition according to the second-order theory developed in the paper.

The effect of axial extensibility (i.e. including the effect of axial strain on the kinematical relationships according to the Reissner model), is analytically quantified and compared to the classical solution of the problem in the following section.

\section{Effect of inclusion of axial extensibility in the kinematical relationships (Reissner theory)}

The specific effect of axial extensibility on the buckling load is investigated in this section. The axial strain in the sub-components of the partial composite beam-column model is included in the kinematics of the problem according to Reissner [47]. This model has been studied by Kryžanowski et al. [56] and Schnabl and Planinc [7], who approximated the nonlinear stability problem by a linearization of the governing equations. This linearized theory of stability is based on the fact that the bifurcation (critical) points of the nonlinear system coincide with the critical points of its equivalent linearized system. It can be shown that the result of the work of Kryžanowski et al. [56] and Schnabl and Planinc [7] on the effect of axial extensibility can be included in the sixth-order differential equation (32) if the following adjustment is made:

$w^{(6)}+\left(\frac{P}{\overline{E I}_{0}}-\alpha^{2}\right) w^{(4)}-\alpha^{2} \frac{P}{\overline{E I}_{\infty}} w^{\prime \prime}=0$

where the extensibility effect is taken into account by replacing the bending stiffness according to the following expressions

$\overline{E I}_{0}=\frac{E I_{0}}{1+\varepsilon} ; \quad \overline{E I}_{\infty}=\frac{E I_{\infty}}{1+\varepsilon} ; \quad$ and $\quad \varepsilon=-\frac{P}{E A_{0}}$.

It is noted that $\overline{E I}_{\infty} / \overline{E I}_{0}=E I_{\infty} / E I_{0}$. It is worth mentioning that $[56,7]$ obtained this differential equation from application of a similar variational principle. This result is evident from the work of Schnabl and Planinc [7], even though they do not explicitly state it themselves, that the extensibility effect can be taken into account by replacing the bending stiffness according to Eq. (66).

The same result was earlier obtained for ordinary columns including the extensibility (and shear) effects by Ziegler [45]. The exact buckling load for a clamped-free partial composite column including this axial extensibility effect $\left(P_{\mathrm{cr}}^{*}\right)$ is then given by

$\frac{P_{\mathrm{cr}}^{*}}{P_{E}^{0}}=\frac{1}{1+\varepsilon} \cdot \frac{\left(\frac{\pi}{2}\right)^{2}+(\alpha L)^{2}}{\left(\frac{\pi}{2}\right)^{2}+\frac{E I_{0}}{E I_{\infty}}(\alpha L)^{2}}$.

If the extensibility effect is neglected $(\varepsilon=0)$, the corresponding buckling load $\left(P_{\mathrm{cr}}\right)$ is given by Eq. (55). Therefore, the ratio between the exact value $P_{\mathrm{cr}}^{*}(\varepsilon \neq 0)$ and the "approximate" one $P_{\mathrm{cr}}$ neglecting the additional extensibility effect $(\varepsilon=0)$ is given by

$\frac{P_{\mathrm{cr}}^{*}}{P_{\mathrm{cr}}}=\frac{1}{1+\varepsilon}>1 \quad$ with $\varepsilon=-\frac{P_{\mathrm{cr}}^{*}}{E A_{0}}$

or equivalently

$\frac{\Delta P_{\mathrm{cr}}}{P_{\mathrm{cr}}^{*}}=\frac{P_{\mathrm{cr}}^{*}-P_{\mathrm{cr}}}{P_{\mathrm{cr}}^{*}}=-\varepsilon>0$.

Note that this formula is identical to the formula associated with the buckling case of a non-composite extensible beam (see for instance $[34,45,37])$. It is generally assumed that this additional extensibility effect can be neglected since the constitutive law 


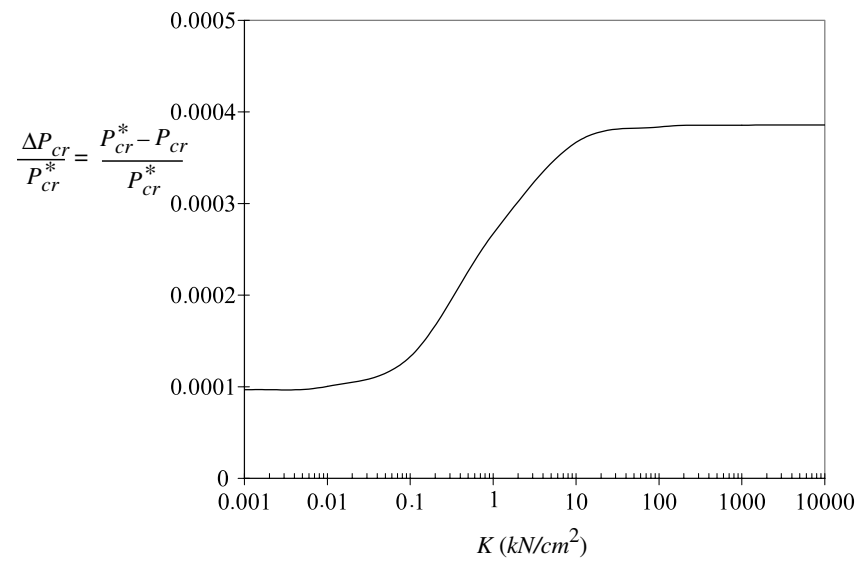

Fig. 10. Effect of axial extensibility on the buckling load of the partial composite Euler-Bernoulli column; $\Delta P_{\mathrm{cr}}=P_{\mathrm{cr}}^{*}-P_{\mathrm{cr}} ; \frac{\Delta P_{\mathrm{cr}}}{P_{\mathrm{cr}}^{*}}=-\varepsilon=-\frac{P_{\mathrm{cr}}^{*}}{E A_{0}}$.

assumes that the strain is small compared to unity (see for instance [35,37]). This last assumption is generally adopted when dealing with buckling of extensible systems as explained, for instance, by Alfutov [35]. This correction increases the buckling load, whereas the shear effect decreases the buckling load. Alfutov [35] discusses the order of magnitude of such a correction with respect to the approximation of the Euler formula. A comparison between both theories can be suggested based on the data used by Girhammar and Pan [2] and Schnabl and Planinc [7]:

$$
\begin{aligned}
& b_{1}=0.3 \mathrm{~m} ; \quad h_{1}=0.05 \mathrm{~m} ; \quad E_{1}=1.2 \times 10^{10} \mathrm{~N} / \mathrm{m}^{2} ; \\
& b_{2}=0.05 \mathrm{~m} ; \quad h_{2}=0.15 \mathrm{~m} ; \\
& E_{2}=0.8 \times 10^{10} \mathrm{~N} / \mathrm{m}^{2} ; \quad L=4 \mathrm{~m} .
\end{aligned}
$$

The subscript " 1 " is referred to the concrete sub-element and " 2 " to the timber sub-element in this example. The buckling loads for non-composite and full composite beam-columns are then given by

$P_{E}^{0}=E I_{0}\left(\frac{\pi}{2 L}\right)^{2}=23131.885 \mathrm{~N}$ and

$P_{E}^{\infty}=E I_{\infty}\left(\frac{\pi}{2 L}\right)^{2}=92527.541 \mathrm{~N}$.

A comparison of the buckling loads for the two theories is presented in Table 1 and Fig. 10 as a function of the slip modulus $K$. For this clamped-free composite column the difference is less than $0.05 \%$. Schnabl and Planinc [7] observe in their study that the difference is less than $0.5 \%$ for most boundary conditions. Axial deformability with respect to the axial strain caused by the internal axial forces is taken into account in most buckling theories of partial composite beam-columns, but this additional extensibility effect related to the change in the deformed geometry of the beam-column caused by the axial strain in the sub-elements as discussed above is generally not taken into account. Neglecting this extensibility is generally a reasonable assumption as is evident from the numerical example treated in Table 1 and illustrated in Fig. 10.

\section{Engesser-Timoshenko theory for partial composite beam- columns-uniform shear model}

Consider a partial composite beam-column where the subelements are modelled according to the Engesser-Timoshenko shear beam-column theory (Fig. 12). The shear deformations are assumed to be equal or what we call uniform in the two subelements. For a beam-column with axially immovable support at
Table 1

Comparison of buckling load values for the Euler-Bernoulli theory without, Eq. (55), and with, Eq. (67), axial extensibility effect for a clamped-free partial composite column.

\begin{tabular}{lll}
\hline$K\left(\mathrm{kN} / \mathrm{cm}^{2}\right)$ & $P_{\text {cr }}(\mathrm{N})$ Eq. $(55)$ & $P_{\mathrm{cr}}^{*}(\mathrm{~N})$ Eq. $(67)$ \\
\hline $10^{-10}$ & 23131.885 & 23134.115 \\
$10^{-5}$ & 23132.886 & 23135.115 \\
$10^{-3}$ & 23231.741 & 23233.991 \\
$10^{-2}$ & 24117.680 & 24120.104 \\
$10^{-1}$ & 31872.371 & 31876.604 \\
1 & 64098.494 & 64115.623 \\
10 & 88024.290 & 88056.598 \\
100 & 92049.284 & 92084.616 \\
1000 & 92479.417 & 92515.080 \\
$10^{5}$ & 92527.060 & 92562.759 \\
$10^{10}$ & 92527.541 & 92563.241 \\
\hline
\end{tabular}

$x=0\left(u_{1}(0)=u_{2}(0)=0\right)$, the energy functional can be expressed as

$$
\begin{aligned}
U\left[u_{1}, u_{2}, w, \psi\right]= & \int_{0}^{L}\left[\frac{1}{2} E_{1} I_{1} \psi^{\prime 2}+\frac{1}{2} \kappa_{1} G_{1} A_{1}\left(w^{\prime}-\psi\right)^{2}\right. \\
& \left.+\frac{1}{2} \overline{N_{1}} w^{\prime 2}+\overline{N_{1}} u_{1}^{\prime}\right] \mathrm{d} x+\int_{0}^{L}\left[\frac{1}{2} E_{2} I_{2} \psi^{\prime 2}\right. \\
& +\frac{1}{2} \kappa_{2} G_{2} A_{2}\left(w^{\prime}-\psi\right)^{2}+\frac{1}{2} \overline{N_{2}} w^{\prime 2} \\
& \left.+\overline{N_{2}} u_{2}^{\prime}+\frac{1}{2} K \cdot \Delta u^{2}\right] \mathrm{d} x \\
& +P_{1} u_{1}(L)+P_{2} u_{2}(L)-\int_{0}^{L} q w \mathrm{~d} x
\end{aligned}
$$

where now the slip is given by

$\Delta u=u_{2}-u_{1}+h_{0} \psi$

where $\psi$ is the rotation of the cross-section of each sub-element from the non-deformed to the deformed state, $\kappa_{i} G_{i} A_{i}$ is the shear stiffness of the $i$ th sub-element $\left(G_{i}\right.$ denotes the shear modulus of the $i$ th sub-element), and $\kappa_{i}$ is the so-called shear coefficient, a dimensionless shape factor depending on the cross-section of the $i$ th sub-element. The underlying assumptions are that the plane crosssections of the sub-elements before deformation remain plane after deformation, but not necessarily perpendicular to the neutral beam axis, and that the shear angle is uniformly distributed over the depth of the cross-section of the sub-element.

The energy functional of ordinary Timoshenko columns (Fig. 11) is well documented in the paper of Bažant [61], Attard and Hunt [16,17], both for the Engesser and the Haringx theories (see also [46]). In the Engesser theory (Fig. 11(a)), the normal force is chosen parallel to the beam axis at the loaded state, whereas in the Haringx approach (Fig. 11(b)), the normal force is chosen normal to the cross-section at the state after loading. The Haringx model (Fig. 11(b)) can be obtained from the Engesser model (Fig. 11(a)) if the shear modulus in the Engesser model is replaced by $G=$ $G-N / A$, i.e. the value of the shear modulus $(G)$ is dependent on the normal stress $(N / A)$ in the cross-section.

The kinematics of the partial composite column according to the Engesser-Timoshenko theory are shown in Fig. 12 for uniform (equal) shear deformation in each sub-element. Applying the stationary condition of the total energy, $\delta U=0$, the following system of differential equations are obtained

$\left\{\begin{array}{l}-E I_{0} \psi^{\prime \prime}-\kappa G A_{0}\left(w^{\prime}-\psi\right)+K h_{0}\left(u_{2}-u_{1}+h_{0} \psi\right)=0 \\ -\kappa G A_{0}\left(w^{\prime \prime}-\psi^{\prime}\right)-\left[\left(\overline{N_{1}}+\overline{N_{2}}\right) w^{\prime}\right]^{\prime}-q=0 \\ -\left(\overline{N_{1}}\right)^{\prime}-K\left(u_{2}-u_{1}+h_{0} \psi\right)=0 \\ -\left(\overline{N_{2}}\right)^{\prime}+K\left(u_{2}-u_{1}+h_{0} \psi\right)=0\end{array}\right.$ 

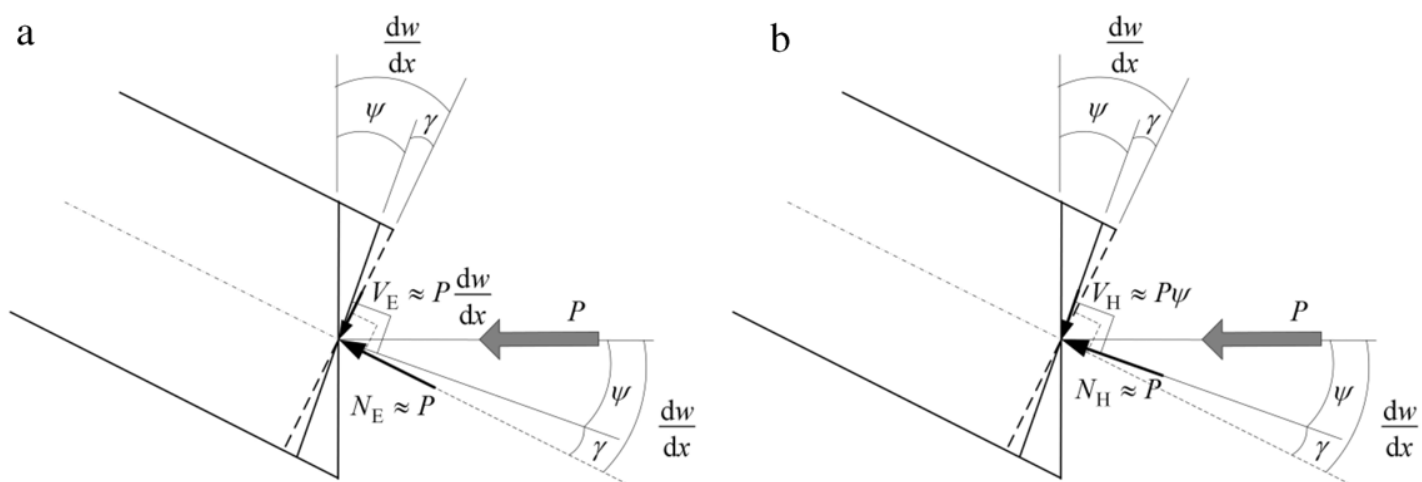

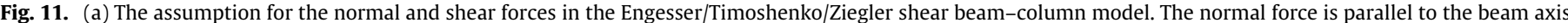

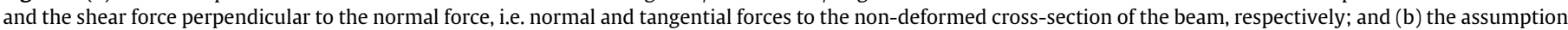

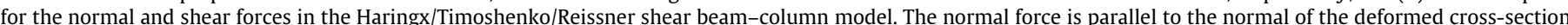

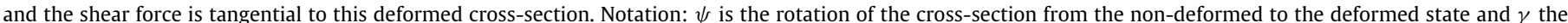
shear angle.

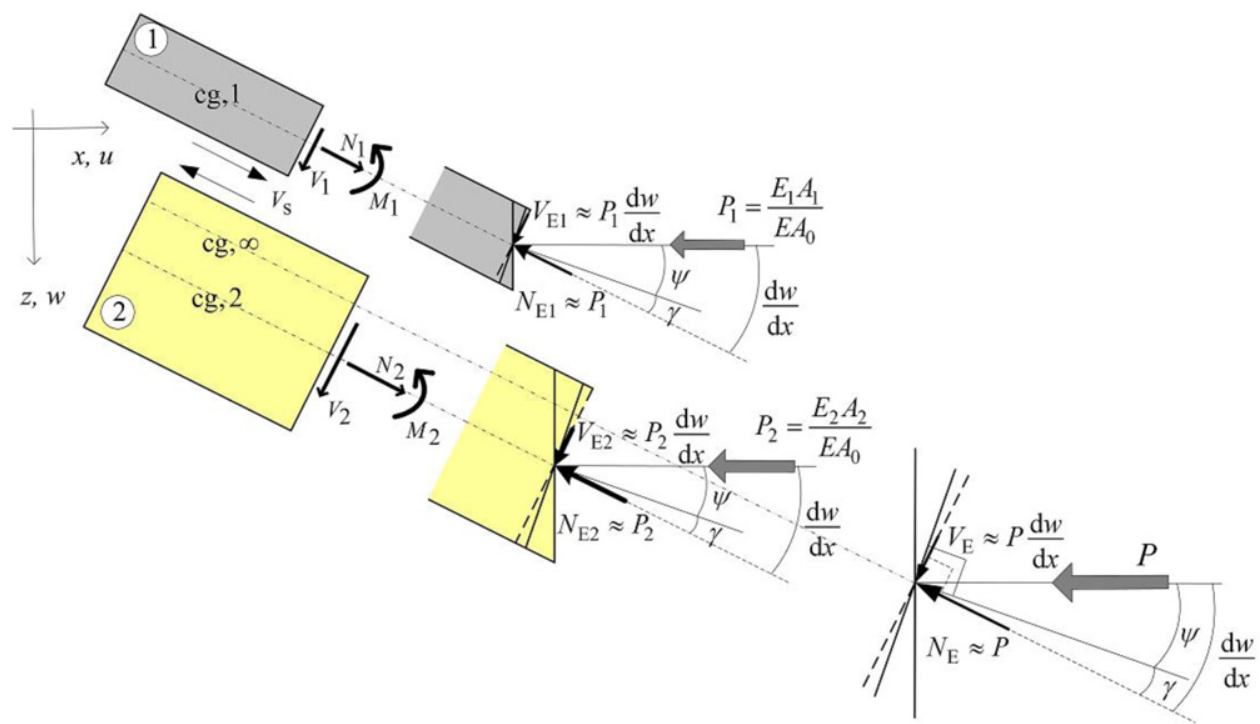

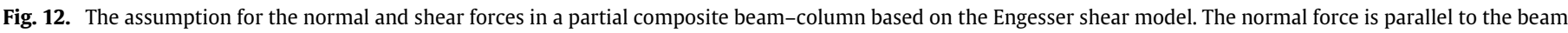
axis and the shear force perpendicular to the normal force, i.e. normal and tangential forces to the non-deformed cross-section of the beam, respectively.

where $\kappa G A_{0}=\kappa_{1} G_{1} A_{1}+\kappa_{2} G_{2} A_{2}$ is the sum of the axial stiffnesses for the two sub-elements. The pertaining following boundary conditions are given by

$\left[E I_{0} \psi^{\prime} \delta \psi\right]_{0}^{L}=0$

$\left[\left(\kappa G A_{0}\left(w^{\prime}-\psi\right)+\left(\overline{N_{1}}+\overline{N_{2}}\right) w^{\prime}\right) \delta w\right]_{0}^{L}=0$

$\left[\overline{N_{1}} \delta u_{1}\right]_{0}^{L}+P_{1} \delta u_{1}(L)=0$

$\left[\overline{N_{2}} \delta u_{2}\right]_{0}^{L}+P_{2} \delta u_{2}(L)=0$.

The third and the fourth differential equations of Eq. (73) lead to the same simplification as given by Eq. (30). The system of differential equations is then reformulated according to the simplified result of Eq. (30):

$$
\left\{\begin{array}{l}
-E I_{0} \psi^{\prime \prime}-\kappa G A_{0}\left(w^{\prime}-\psi\right)+K h_{0}\left(u_{2}-u_{1}+h_{0} \psi\right)=0 \\
-\kappa G A_{0}\left(w^{\prime \prime}-\psi^{\prime}\right)+P w^{\prime \prime}-q=0 \\
-E_{1} A_{1} u_{1}^{\prime \prime}-K\left(u_{2}-u_{1}+h_{0} \psi\right)=0 \\
-E_{2} A_{2} u_{2}^{\prime \prime}+K\left(u_{2}-u_{1}+h_{0} \psi\right)=0
\end{array}\right.
$$

with the following boundary conditions:

$\left[E I_{0} \psi^{\prime} \delta \psi\right]_{0}^{L}=0$

$\left[\left(\kappa G A_{0}\left(w^{\prime}-\psi\right)-P w^{\prime}\right) \delta w\right]_{0}^{L}=0$

$$
\begin{array}{ll}
u_{1}(0)=0 ; & E_{1} A_{1} u_{1}^{\prime}(L)=-P_{1} \\
u_{2}(0)=0 ; & E_{2} A_{2} u_{2}^{\prime}(L)=-P_{2} .
\end{array}
$$

After some mathematical manipulations, a sixth-order differential equation can be obtained for the deflection:

$$
\begin{aligned}
& \left(1-\frac{P}{\kappa G A_{0}}\right) w^{(6)}+\left[\frac{P}{E I_{0}}-\alpha^{2}\left(1-\frac{P}{\kappa G A_{0}}\right)\right] w^{(4)} \\
& -\alpha^{2} \frac{P}{E I_{\infty}} w^{\prime \prime}=-\frac{q^{(4)}}{\kappa G A_{0}}+\frac{q^{\prime \prime}}{E I_{0}}\left(1+\frac{\alpha^{2} E I_{0}}{\kappa G A_{0}}\right)-\frac{\alpha^{2}}{E I_{\infty}} q .
\end{aligned}
$$

This differential equation has already been derived by $\mathrm{Xu}$ and $\mathrm{Wu}$ [6], but by using direct equilibrium arguments. The advantage of using the variational method as in this paper is that it also will render the well-posed physical boundary conditions. These results using a energy formulation, the governing differential equation and the pertaining admissible natural boundary conditions, have not been published before in the literature. The differential equation (31) is found again as a particular case when $\kappa G A_{0} \rightarrow$ $\infty$ (Euler-Bernoulli beam-columns with partial interaction). The buckling mode is also assumed in the same format as that given in Eq. (54) and the resulting buckling load for the Engesser model is 


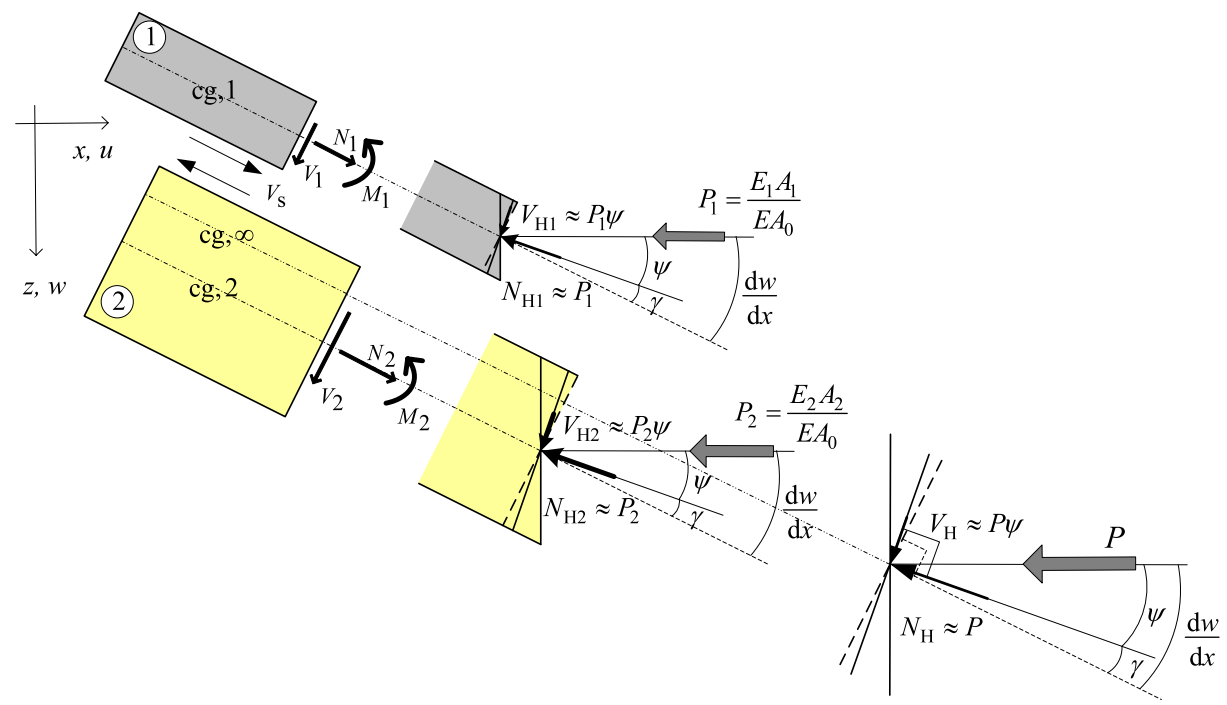

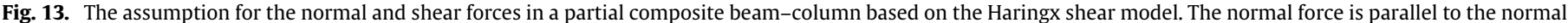

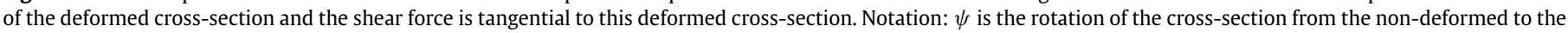
deformed state and $\gamma$ the shear angle.

Table 2

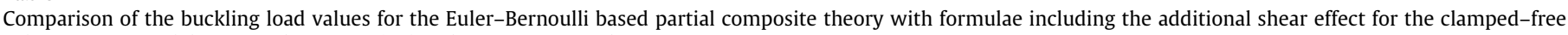
column. Numerical data according to Eq. (70) and $E_{1} / G_{1}=2.4$ and $E_{2} / G_{2}=16$.

\begin{tabular}{|c|c|c|c|c|c|}
\hline$K\left(\mathrm{kN} / \mathrm{cm}^{2}\right)$ & $P_{\text {cr }}(\mathrm{N})$ Euler-Bernoulli & $P_{\mathrm{cr}}^{*}(\mathrm{~N})$ Engesser $\psi_{1}=\psi_{2}$ & $P_{\mathrm{cr}}^{*}(\mathrm{~N})$ Haringx $\psi_{1}=\psi_{2}$ & $P_{\mathrm{cr}}^{*}(\mathrm{~N})$ Engesser $\psi_{1} \neq \psi_{2}$ & $P_{\mathrm{cr}}^{*}(\mathrm{~N})$ Haringx $\psi_{1} \neq \psi_{2}$ \\
\hline $10^{-10}$ & 23131.885 & 23123.735 & 23123.737 & 23035.567 & 23035.742 \\
\hline $10^{-5}$ & 23132.886 & 23124.734 & 23124.737 & 23036.559 & 23036.734 \\
\hline $10^{-3}$ & 23231.741 & 23223.520 & 23223.523 & 23134.591 & 23134.769 \\
\hline $10^{-2}$ & 24117.680 & 24108.820 & 24108.823 & 24012.996 & 24013.194 \\
\hline $10^{-1}$ & 31872.371 & 31856.898 & 31856.906 & 31689.797 & 31690.255 \\
\hline 1 & 64098.494 & 64035.948 & 64036.009 & 63364.332 & 63367.971 \\
\hline 10 & 88024.290 & 87906.379 & 87906.537 & 86645.657 & 86654.944 \\
\hline 100 & 92049.284 & 91920.352 & 91920.532 & 90542.769 & 90553.363 \\
\hline 1000 & 92479.417 & 92349.278 & 92349.460 & 90958.906 & 90969.646 \\
\hline $10^{5}$ & 92527.060 & 92396.786 & 92396.969 & 91004.994 & 91015.751 \\
\hline $10^{10}$ & 92527.541 & 92397.266 & 92397.449 & 91005.460 & 91016.217 \\
\hline
\end{tabular}

then given by

$$
\frac{P_{\mathrm{cr}}^{*}}{P_{E}^{0}}=\frac{\left(\frac{\pi}{2}\right)^{2}+(\alpha L)^{2}}{\left(\frac{\pi}{2}\right)^{2}+\frac{E I_{0}}{E I_{\infty}}(\alpha L)^{2}+\frac{E I_{0}}{\kappa G A_{0}}\left(\frac{\pi}{2 L}\right)^{2}\left[\left(\frac{\pi}{2}\right)^{2}+(\alpha L)^{2}\right]}
$$

where the buckling length coefficient " 2 " in the term $(\pi / 2 L)^{2}$ represents the cantilever case. For infinite shear stiffness $\left(\kappa G A_{0} \rightarrow\right.$ $\infty)$, Eq. (78) is reduced to Eq. (55). Finally, the effect of shear deformation on the buckling load can be evaluated by relating the buckling load $\left(P_{\mathrm{cr}}^{*}\right)$ according to Eq. $(78)$ to the buckling load $\left(P_{\mathrm{cr}}\right)$ according to Eq. (55) (Euler-Bernoulli column theory) to give

$\frac{P_{\mathrm{cr}}^{*}}{P_{\mathrm{cr}}}=\frac{1}{1+\frac{P_{\mathrm{cr}}}{\kappa G A_{0}}}$.

Eq. (79) is the generalized Engesser formulae for partial composite Timoshenko columns. It is worth mentioning that Engesser formulae for the solid Timoshenko column is valid for most boundary conditions, however, it is not generally applicable, for instance, for the fixed-pinned conditions (see $[10,45,62])$. The same conclusion with respect to the fixed-pinned boundary conditions was noticed by Girhammar and Pan [2] for partial composite Euler-Bernoulli beam-column theory. The same remark is also valid for the Bickford-Reddy ordinary column buckling (see [55]) and for the composite Timoshenko beam-column model with partial interaction.

For a specific numerical example as discussed in the next section, the results for the Engesser model (together with the other models discussed in this paper) are presented in Table 2 and Fig. 14. For further details, the reader is referred to Section 8 .

\section{Haringx-Timoshenko theory for partial composite beam-columns-uniform shear model}

Consider a partial composite beam-column where the subelements are modelled according to the Haringx-Timoshenko shear beam-column theory (Fig. 13) with uniform (equal) shear deformations in the two sub-elements. For a beam-column with axially immovable support at $x=0\left(u_{1}(0)=u_{2}(0)=0\right)$, the energy functional can be expressed as

$$
\begin{aligned}
& U\left[u_{1}, u_{2}, w, \psi\right] \\
& =\int_{0}^{L} \frac{1}{2} E_{1} I_{1} \psi^{\prime 2}+\frac{1}{2}\left(\kappa_{1} G_{1} A_{1}-\overline{N_{1}}\right)\left(w^{\prime}-\psi\right)^{2} \\
& \quad+\frac{1}{2} \overline{N_{1}} w^{\prime 2}+\overline{N_{1}} u_{1}^{\prime} \mathrm{d} x+\int_{0}^{L} \frac{1}{2} E_{2} I_{2} \psi^{\prime 2} \\
& \quad+\frac{1}{2}\left(\kappa_{2} G_{2} A_{2}-\overline{N_{2}}\right)\left(w^{\prime}-\psi\right)^{2}+\frac{1}{2} \overline{N_{2}} w^{\prime 2}+\overline{N_{2}} u_{2}^{\prime} \\
& \quad+\frac{1}{2} K \cdot \Delta u^{2} \mathrm{~d} x+P_{1} u_{1}(L)+P_{2} u_{2}(L)-\int_{0}^{L} q w \mathrm{~d} x
\end{aligned}
$$

where the slip $(\Delta u)$ is given by Eq. (72b). Using the same procedure as in Section 7, the following system of differential equations are 
obtained

$$
\left\{\begin{array}{l}
-E I_{0} \psi^{\prime \prime}-\left(\kappa G A_{0}-\overline{N_{1}}-\overline{N_{2}}\right)\left(w^{\prime}-\psi\right) \\
\quad+K h_{0}\left(u_{2}-u_{1}+h_{0} \psi\right)=0 \\
-\kappa G A_{0}\left(w^{\prime \prime}-\psi^{\prime}\right)-\left[\left(\overline{N_{1}}+\overline{N_{2}}\right) \psi\right]^{\prime}-q=0 \\
-\left(\overline{N_{1}}\right)^{\prime}-K\left(u_{2}-u_{1}+h_{0} \psi\right)=0 \\
-\left(\overline{N_{2}}\right)^{\prime}+K\left(u_{2}-u_{1}+h_{0} \psi\right)=0
\end{array}\right.
$$

with the following boundary conditions:

$$
\begin{aligned}
& {\left[E I_{0} \psi^{\prime} \delta \psi\right]_{0}^{L}=0} \\
& {\left[\left(\kappa G A_{0}\left(w^{\prime}-\psi\right)+\left(\overline{N_{1}}+\overline{N_{2}}\right) \psi\right) \delta w\right]_{0}^{L}=0} \\
& {\left[\overline{N_{1}} \delta u_{1}\right]_{0}^{L}+P_{1} \delta u_{1}(L)=0} \\
& {\left[\overline{N_{2}} \delta u_{2}\right]_{0}^{L}+P_{2} \delta u_{2}(L)=0 .}
\end{aligned}
$$

The third and the fourth differential equations of Eq. (81) lead to the same simplification as given by Eq. (30). The system of differential equation (81) can be then reformulated as

$$
\left\{\begin{array}{c}
-E I_{0} \psi^{\prime \prime}-\left(\kappa G A_{0}+P\right)\left(w^{\prime}-\psi\right) \\
+K h_{0}\left(u_{2}-u_{1}+h_{0} \psi\right)=0 \\
-\kappa G A_{0} w^{\prime \prime}+\left(\kappa G A_{0}+P\right) \psi^{\prime}-q=0 \\
-E_{1} A_{1} u_{1}^{\prime \prime}-K\left(u_{2}-u_{1}+h_{0} \psi\right)=0 \\
-E_{2} A_{2} u_{2}^{\prime \prime}+K\left(u_{2}-u_{1}+h_{0} \psi\right)=0
\end{array}\right.
$$

together with the following boundary conditions:

$$
\begin{aligned}
& {\left[E I_{0} \psi^{\prime} \delta \psi\right]_{0}^{L}=0} \\
& {\left[\left(\kappa G A_{0} w^{\prime}-\left(\kappa G A_{0}+P\right) \psi\right) \delta w\right]_{0}^{L}=0} \\
& u_{1}(0)=0 ; \quad E_{1} A_{1} u_{1}^{\prime}(L)=-P_{1} \\
& u_{2}(0)=0 ; \quad E_{2} A_{2} u_{2}^{\prime}(L)=-P_{2} .
\end{aligned}
$$

The corresponding sixth-order differential equation for the Haringx theory can then be obtained for the deflection as

$$
\begin{aligned}
& \frac{\kappa G A_{0}}{\kappa G A_{0}+P} w^{(6)}+\left[\frac{P}{E I_{0}}-\alpha^{2} \frac{\kappa G A_{0}}{\kappa G A_{0}+P}\right] w^{(4)}-\alpha^{2} \frac{P}{E I_{\infty}} w^{\prime \prime} \\
& =-\frac{q^{(4)}}{\kappa G A_{0}+P}+\frac{q^{\prime \prime}}{E I_{0}}\left(1+\frac{\alpha^{2} E I_{0}}{\kappa G A_{0}+P}\right)-\frac{\alpha^{2}}{E I_{\infty}} q .
\end{aligned}
$$

This differential equation has not previously been derived to the authors' knowledge. The differential equation (31) is found again as a particular case when $\kappa G A_{0} \rightarrow \infty$ (Euler-Bernoulli beam-columns with partial interaction). Using the buckling mode according to Eq. (54), a second-order polynomial equation for the Haringx buckling load is obtained, which gives:

$$
\begin{aligned}
\frac{P_{\mathrm{cr}}^{*}}{P_{E}^{0}}= & \frac{1}{2} \frac{\kappa G A_{0}}{E I_{0}}\left(\frac{2 L}{\pi}\right)^{2} \\
& \times\left[-1+\sqrt{1+4 \frac{E I_{0}}{\kappa G A_{0}}\left(\frac{\pi}{2 L}\right)^{2} \frac{\left(\frac{\pi}{2}\right)^{2}+(\alpha L)^{2}}{\left(\frac{\pi}{2}\right)^{2}+\frac{E I_{0}}{E I_{\infty}}(\alpha L)^{2}}}\right] .
\end{aligned}
$$

With reference to the buckling load $\left(P_{\mathrm{cr}}\right)$ given by Eq. (55), the Haringx buckling load $\left(P_{\mathrm{cr}}^{*}\right)$ can be expressed as

$P_{\mathrm{cr}}^{*}=\frac{\kappa G A_{0}}{2}\left(-1+\sqrt{1+4 \frac{P_{\mathrm{cr}}}{\kappa G A_{0}}}\right)$.

Eq. (87) is the generalized Haringx formulae for composite Timoshenko columns with partial interaction. In both cases, Engesser and Haringx type of formula, the shear effect decreases the buckling load. For sufficiently large columns, i.e. $P_{\mathrm{cr}}<<\kappa G A_{0}$, the shear effect can be neglected. For instance, for the numerical

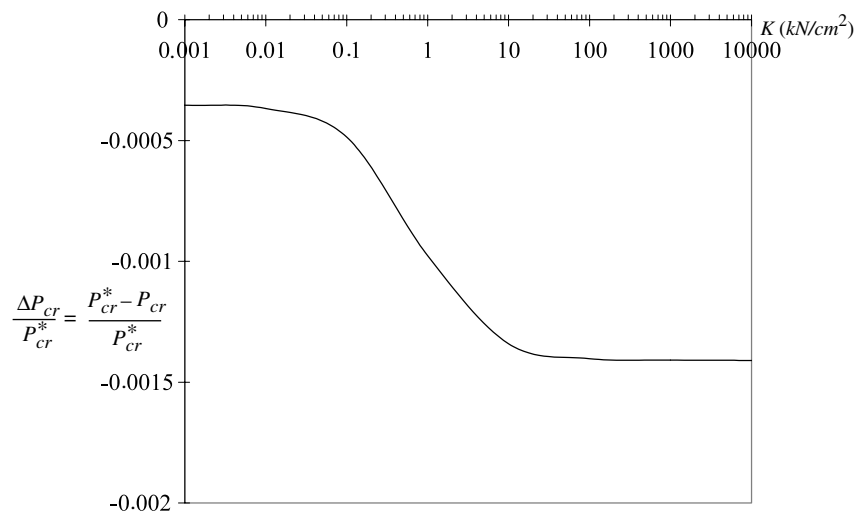

Fig. 14. Effect of shear deformation on the buckling load of a partial composite Timoshenko column based on the Engesser uniform shear theory; $\Delta P_{\mathrm{cr}}=P_{\mathrm{cr}}^{*}-$ $P_{\mathrm{cr}} ; \frac{\Delta P_{\mathrm{cr}}}{P_{\mathrm{cr}}^{*}}=-\frac{P_{\mathrm{cr}}}{\kappa G A_{0}} \leq 0$.

example previously discussed according to Eq. (70), the shear effect can be taken into account using the following parameters:

$\frac{E_{1}}{G_{1}}=2.4$ and $\frac{E_{2}}{G_{2}}=16$.

The shape factors for a rectangular beam is given by (see for instance [65]):

$\kappa_{1}=\frac{5}{6}$ and $\kappa_{2}=\frac{5}{6}$.

A comparison between the different theories by Euler-Bernoulli, Engesser and Haringx applied to clamped-free partial composite columns is presented in Table 2 and Fig. 14, at this point for uniform shear $\left(\psi_{1}=\psi_{2}\right)$. It is obvious that in this case the shear effect can be neglected, the difference is lower than $0.2 \%$ (cf. Fig. 14). It is also evident that the numerical values for the Haringx based theory are very close to those for the Engesser based theory. For convenience, only the results for the Engesser case is reported in Fig. 14, but the corresponding Haringx curve can be superposed on the Engesser one in this figure. For short columns or composite built-up columns, however, the shear effect should be included. The shear effect can also be important to incorporate in the analysis for columns where the core material or the interlayer is soft in shear, for example for high ratios between the Young modulus $E_{i}$ and the shear modulus $G_{i}$ of the sub-elements.

\section{Engesser-Timoshenko theory for partial composite beam- columns-differential shear model}

\subsection{Variational formulation of the buckling problem}

As a main assumption of the composite Timoshenko column theories presented in the previous Sections 7 and 8, the shear deformation was assumed to be the same in all layers. However, a more realistic model is to assume different shear deformations or as we call it differential shear in the different sub-elements, see Fig. 15 where the kinematics is illustrated. Such a model with this kinematical assumption has already been implemented by Schnabl et al. [57] in case of bending of partial composite Timoshenko beams. In this section, we will develop some analytical solutions associated with the buckling of partial composite Timoshenko columns including this differential shear effect.

For an Engesser-Timoshenko beam-column with axially immovable support at $x=0\left(u_{1}(0)=u_{2}(0)=0\right)$, the energy functional can be expressed as 


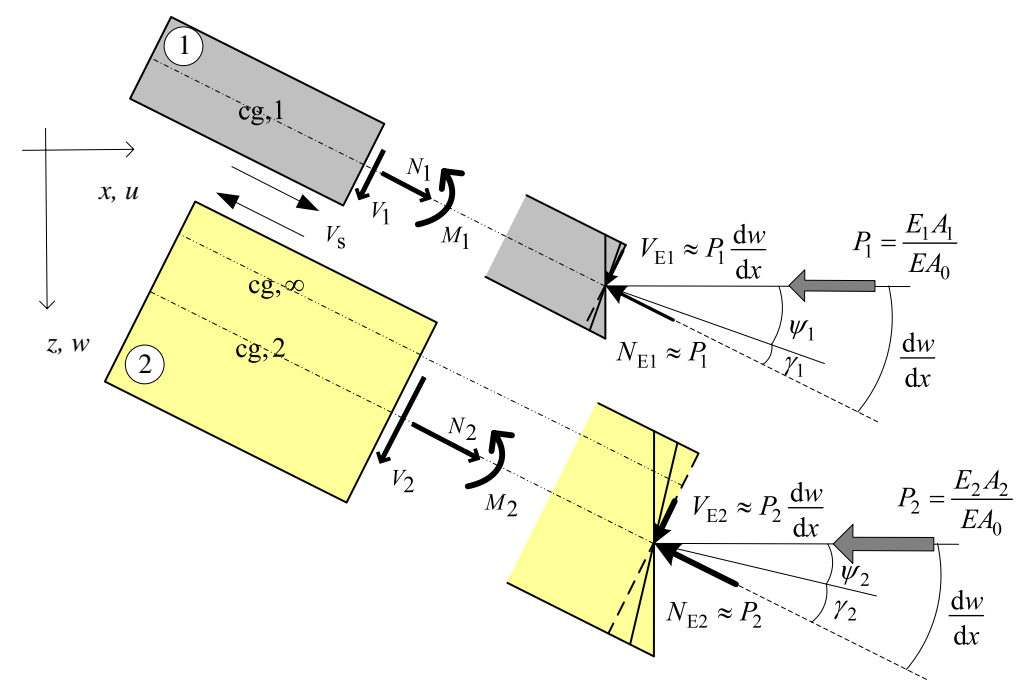

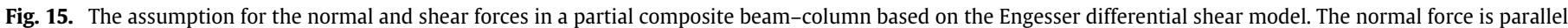
to the beam axis and the shear force perpendicular to the normal force, i.e. normal and tangential forces to the non-deformed cross-section of the beam, respectively.

$U\left[u_{1}, u_{2}, w, \psi_{1}, \psi_{2}\right]$

$$
\begin{aligned}
= & \int_{0}^{L}\left[\frac{1}{2} E_{1} I_{1} \psi_{1}^{\prime 2}+\frac{1}{2} \kappa_{1} G_{1} A_{1}\left(w^{\prime}-\psi_{1}\right)^{2}\right. \\
& \left.+\frac{1}{2} \overline{N_{1}} w^{\prime 2}+\overline{N_{1}} u_{1}^{\prime}\right] \mathrm{d} x+\int_{0}^{L}\left[\frac{1}{2} E_{2} I_{2} \psi_{2}^{\prime 2}\right. \\
& +\frac{1}{2} \kappa_{2} G_{2} A_{2}\left(w^{\prime}-\psi_{2}\right)^{2}+\frac{1}{2} \overline{N_{2}} w^{\prime 2}+\overline{N_{2}} u_{2}^{\prime} \\
& \left.+\frac{1}{2} K \cdot \Delta u^{2}\right] \mathrm{d} x+P_{1} u_{1}(L)+P_{2} u_{2}(L)-\int_{0}^{L} q w \mathrm{~d} x
\end{aligned}
$$

where now the slip is expressed as

$\Delta u=u_{2}-u_{1}+\frac{h_{1}}{2} \psi_{1}+\frac{h_{2}}{2} \psi_{2}$.

For uniform shear $\left(\psi_{1}=\psi_{2}\right)$, Eq. (90) is reduced to Eq. (72). Applying the stationary condition of the total energy, $\delta U=0$, the following system of differential equations are obtained

$$
\left\{\begin{array}{l}
-E_{1} I_{1} \psi_{1}^{\prime \prime}-\kappa_{1} G_{1} A_{1}\left(w^{\prime}-\psi_{1}\right) \\
+K \frac{h_{1}}{2}\left(u_{2}-u_{1}+\frac{h_{1}}{2} \psi_{1}+\frac{h_{2}}{2} \psi_{2}\right)=0 \\
-E_{2} I_{2} \psi_{2}^{\prime \prime}-\kappa_{2} G_{2} A_{2}\left(w^{\prime}-\psi_{2}\right) \\
\quad+K \frac{h_{2}}{2}\left(u_{2}-u_{1}+\frac{h_{1}}{2} \psi_{1}+\frac{h_{2}}{2} \psi_{2}\right)=0 \\
-\kappa_{1} G_{1} A_{1}\left(w^{\prime \prime}-\psi_{1}^{\prime}\right)-\kappa_{2} G_{2} A_{2}\left(w^{\prime \prime}-\psi_{2}^{\prime}\right) \\
-\left[\left(\overline{N_{1}}+\overline{N_{2}}\right) w^{\prime}\right]^{\prime}-q=0 \\
-\left(\overline{N_{1}}\right)^{\prime}-K\left(u_{2}-u_{1}+\frac{h_{1}}{2} \psi_{1}+\frac{h_{2}}{2} \psi_{2}\right)=0 \\
-\left(\overline{N_{2}}\right)^{\prime}+K\left(u_{2}-u_{1}+\frac{h_{1}}{2} \psi_{1}+\frac{h_{2}}{2} \psi_{2}\right)=0
\end{array}\right.
$$

with the following boundary conditions:

$$
\begin{aligned}
& {\left[E_{1} I_{1} \psi_{1}^{\prime} \delta \psi_{1}\right]_{0}^{L}=0} \\
& {\left[E_{2} I_{2} \psi_{2}^{\prime} \delta \psi_{2}\right]_{0}^{L}=0} \\
& {\left[\left(\kappa_{1} G_{1} A_{1}\left(w^{\prime}-\psi_{1}\right)+\kappa_{2} G_{2} A_{2}\left(w^{\prime}-\psi_{2}\right)\right.\right.} \\
& \left.\left.\quad+\left(\overline{N_{1}}+\overline{N_{2}}\right) w^{\prime}\right) \delta w\right]_{0}^{L}=0 \\
& {\left[\overline{N_{1}} \delta u_{1}\right]_{0}^{L}+P_{1} \delta u_{1}(L)=0} \\
& {\left[\overline{N_{2}} \delta u_{2}\right]_{0}^{L}+P_{2} \delta u_{2}(L)=0 .}
\end{aligned}
$$

The fourth and the fifth differential equations of Eq. (91) lead to the same simplification as given by Eq. (30). The system of differential equations is then reformulated as:

$$
\left\{\begin{array}{c}
-E_{1} I_{1} \psi_{1}^{\prime \prime}-\kappa_{1} G_{1} A_{1}\left(w^{\prime}-\psi_{1}\right) \\
+K \frac{h_{1}}{2}\left(u_{2}-u_{1}+\frac{h_{1}}{2} \psi_{1}+\frac{h_{2}}{2} \psi_{2}\right)=0 \\
-E_{2} I_{2} \psi_{2}^{\prime \prime}-\kappa_{2} G_{2} A_{2}\left(w^{\prime}-\psi_{2}\right) \\
+K \frac{h_{2}}{2}\left(u_{2}-u_{1}+\frac{h_{1}}{2} \psi_{1}+\frac{h_{2}}{2} \psi_{2}\right)=0 \\
-\kappa_{1} G_{1} A_{1}\left(w^{\prime \prime}-\psi_{1}^{\prime}\right)-\kappa_{2} G_{2} A_{2}\left(w^{\prime \prime}-\psi_{2}^{\prime}\right) \\
\quad+P w^{\prime \prime}-q=0 \\
-E_{1} A_{1} u_{1}^{\prime \prime}-K\left(u_{2}-u_{1}+\frac{h_{1}}{2} \psi_{1}+\frac{h_{2}}{2} \psi_{2}\right)=0 \\
-E_{2} A_{2} u_{2}^{\prime \prime}+K\left(u_{2}-u_{1}+\frac{h_{1}}{2} \psi_{1}+\frac{h_{2}}{2} \psi_{2}\right)=0
\end{array}\right.
$$

with the following boundary conditions:

$\left[E_{1} I_{1} \psi_{1}^{\prime} \delta \psi_{1}\right]_{0}^{L}=0$

$\left[E_{2} I_{2} \psi_{2}^{\prime} \delta \psi_{2}\right]_{0}^{L}=0$

$\left[\left(\kappa_{1} G_{1} A_{1}\left(w^{\prime}-\psi_{1}\right)+\kappa_{2} G_{2} A_{2}\left(w^{\prime}-\psi_{2}\right)-P w^{\prime}\right) \delta w\right]_{0}^{L}=0$

$u_{1}(0)=0 ; \quad E_{1} A_{1} u_{1}^{\prime}(L)=-P_{1}$

$u_{2}(0)=0 ; \quad E_{2} A_{2} u_{2}^{\prime}(L)=-P_{2}$.

9.2. Simplified buckling solution for a double symmetrical crosssection

The case with two identical sub-elements will first be treated as a particular case. Such a situation is usually met in timber engineering, where layered wood beams with two identical subelements are used. In this case, the geometrical and material parameters are reduced to:

$E_{1} I_{1}=E_{2} I_{2}=\frac{E I_{0}}{2}$

$\kappa_{1} G_{1} A_{1}=\kappa_{2} G_{2} A_{2}=\frac{\kappa G A_{0}}{2} ; \quad$ and $\quad h_{1}=h_{2}=h_{0}$.

Subtracting the first and second differential equation in Eq. (93) leads to the following second-order differential equation:

$E I_{0}\left(\psi_{1}-\psi_{2}\right)^{\prime \prime}-\kappa G A_{0}\left(\psi_{1}-\psi_{2}\right)=0$ 


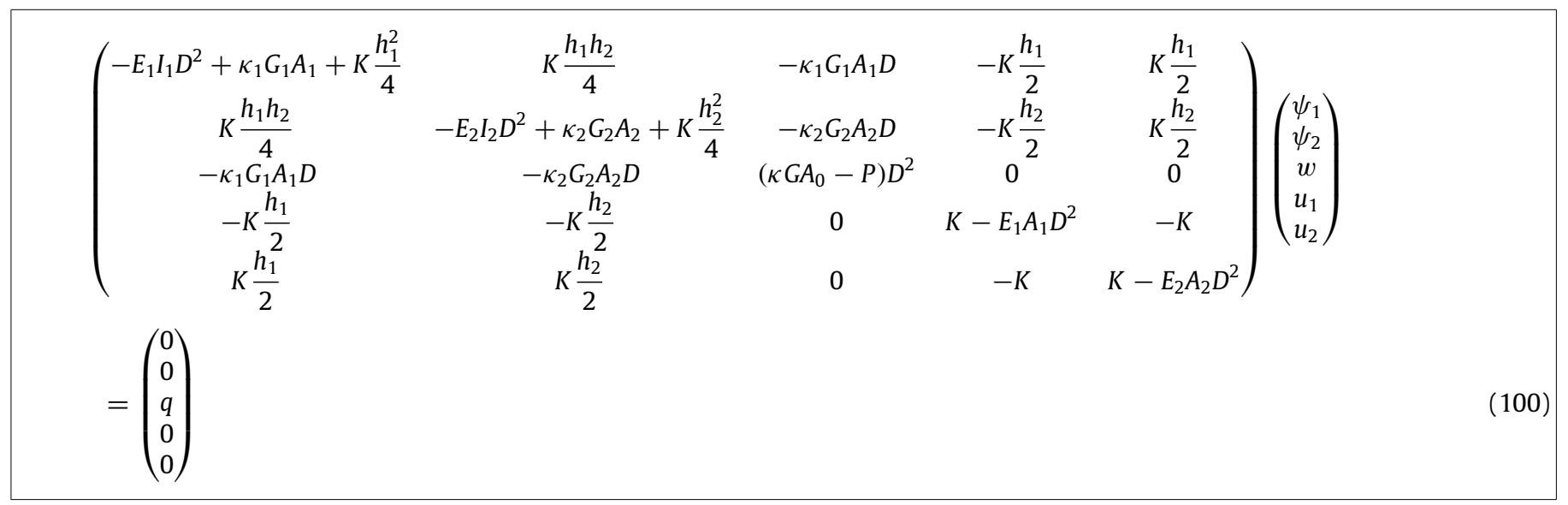

Box I.

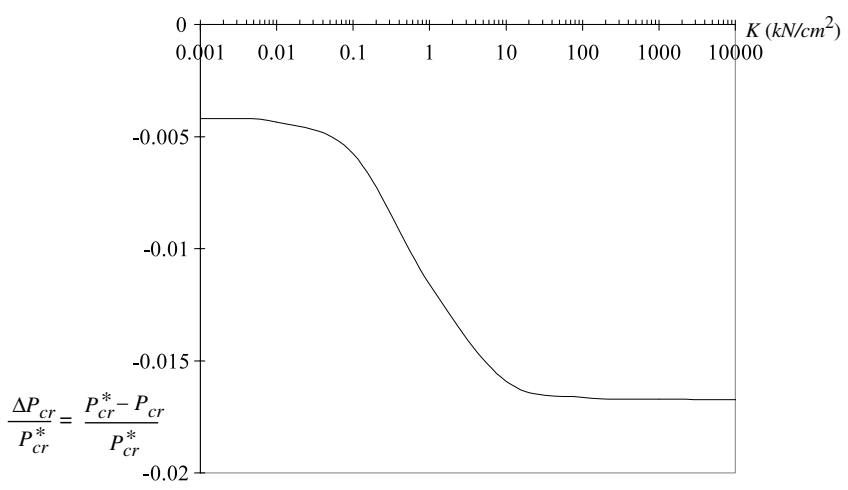

Fig. 16. Effect of shear deformation on the buckling load of a partial composite Timoshenko column based on the Engesser differential shear theory; $\Delta P_{\mathrm{cr}}=P_{\mathrm{cr}}^{*}-$ $P_{\mathrm{cr}} ; P_{\mathrm{cr}}^{*}$, calculated from Eq. (102).

with the following boundary conditions for each sub-element

$\left[\frac{E I_{0}}{2} \psi_{1}^{\prime} \delta \psi_{1}\right]_{0}^{L}=0$ and $\left[\frac{E I_{0}}{2} \psi_{2}^{\prime} \delta \psi_{2}\right]_{0}^{L}=0$.

Assuming symmetry for the boundary conditions for each subelement of the composite beam-column, the boundary conditions (97) can be presented in the following synthetic form:

$\left[\frac{E I_{0}}{2}\left(\psi_{1}^{\prime}-\psi_{2}^{\prime}\right) \delta\left(\psi_{1}-\psi_{2}\right)\right]_{0}^{L}=0$.

In this double symmetrical case with the symmetrical boundary conditions (98) expressed in terms of the difference $\psi_{1}-\psi_{2}$, Eq. (96) is a linear second-order differential equation expressed in this difference variable. The solution of this mathematical problem is simply the uniform shear solution:

$\psi_{1}-\psi_{2}=0$.

This means that in this case, the differential shear model for the Timoshenko column gives exactly the same results as that of the uniform shear model. This conclusion leads to the same buckling load solution for partial composite Engesser-Timoshenko column according to Eq. (79).

\subsection{Buckling solution for a general cross-section}

The differential equations (93) can be presented in a differential matrix format as given in Box I, where $D=\mathrm{d} / \mathrm{d} x$ is the differential operator. The buckling solution based on the second-order theory without lateral loading $(q=0)$ is found from the determinant of the $5 \times 5$ matrix (see Box II) applied to the generic variable $X=\psi_{1}, \psi_{2}, w, u_{1}$ or $u_{2}$.

The differential equation system (101) can be combined into a single tenth-order differential equation satisfied by $[X]$. The buckling solution for the partial composite fixed-free Timoshenko column, is obtained by inserting the buckling shape given in Eq. (54) into the differential equation (101), and is given by Eq. (102) in Box III, where $j^{2}=-1$ ( $j$ is the imaginary number). The numerical values for the buckling solution (102) are given in Table 2 for differential shear $\left(\psi_{1} \neq \psi_{2}\right)$. This solution is compared with the corresponding solutions for the uniform shear model and the Euler-Bernoulli model without shear effects. In this case (unsymmetrical case), the shear effect of the differential model is more pronounced than for the uniform shear model. The specific shear effect decreases the buckling load up to $2 \%$ compared to the Euler-Bernoulli buckling load (see Fig. 16). This difference is still small, but the shear effects can be more significant for shorter columns, for columns soft in shear and columns with other boundary conditions.

In the double symmetrical case, the determinant in Eq. (101) is written in the form given in Box IV.

In this case, the tenth-order differential equation can be factorized in the following way:

$$
\begin{aligned}
& E A_{0} D^{2}\left(E I_{0} D^{2}-\kappa G A_{0}\right)\left[\left(1-\frac{P}{\kappa G A_{0}}\right) D^{6}\right. \\
& \left.+\left[\frac{P}{E I_{0}}-\alpha^{2}\left(1-\frac{P}{\kappa G A_{0}}\right)\right] D^{4}-\alpha^{2} \frac{P}{E I_{\infty}} D^{2}\right][X]=0
\end{aligned}
$$

with $\alpha^{2}=K\left(\frac{h_{0}^{2}}{E I_{0}}+\frac{4}{E A_{0}}\right)=K \frac{4 E I_{\infty}}{E A_{0} E I_{0}}$.

The sixth-order differential equation (77) is clearly recognized in Eq. (104) when the deflection function $X=w$ is considered. In other words, in the double symmetrical case, the tenth-order differential equation of the differential shear Timoshenko model is reduced into a sixth-order differential equation, a fact also observed for the out-of-plane behaviour of partial composite Euler-Bernoulli beams [31].

As a main conclusion, the uniform and differential shear models for partial composite beam-columns are equivalent for double symmetrical beam-columns with material symmetry and with symmetrical boundary conditions (conditions typical for layered wood beams with two identical sub-elements). For general composite beam-columns discussed in this paper, the shear effect should be included using the differential shear model as developed in this section. 


$$
\left|\begin{array}{ccccc}
-E_{1} I_{1} D^{2}+\kappa_{1} G_{1} A_{1}+K \frac{h_{1}^{2}}{4} & K \frac{h_{1} h_{2}}{4} & -\kappa_{1} G_{1} A_{1} D & -K \frac{h_{1}}{2} & K \frac{h_{1}}{2} \\
K \frac{h_{1} h_{2}}{4} & -E_{2} I_{2} D^{2}+\kappa_{2} G_{2} A_{2}+K \frac{h_{2}^{2}}{4} & -\kappa_{2} G_{2} A_{2} D & -K \frac{h_{2}}{2} & K \frac{h_{2}}{2} \\
-\kappa_{1} G_{1} A_{1} D & -\kappa_{2} G_{2} A_{2} D & \left(\kappa G A_{0}-P\right) D^{2} & 0 & 0 \\
-K \frac{h_{1}}{2} & -K \frac{h_{2}}{2} & 0 & K-E_{1} A_{1} D^{2} & -K \\
K \frac{h_{1}}{2} & K \frac{h_{2}}{2} & 0 & -K & K-E_{2} A_{2} D^{2}
\end{array}\right|[X]=0
$$

Box II.

$$
\left|\begin{array}{ccccc}
E_{1} I_{1} \frac{\pi^{2}}{4 L^{2}}+\kappa_{1} G_{1} A_{1}+K \frac{h_{1}^{2}}{4} & K \frac{h_{1} h_{2}}{4} & -j \kappa_{1} G_{1} A_{1} \frac{\pi}{2 L} & -K \frac{h_{1}}{2} & K \frac{h_{1}}{2} \\
K \frac{h_{1} h_{2}}{4} & E_{2} I_{2} \frac{\pi^{2}}{4 L^{2}}+\kappa_{2} G_{2} A_{2}+K \frac{h_{2}^{2}}{4} & -j \kappa_{2} G_{2} A_{2} \frac{\pi}{2 L} & -K \frac{h_{2}}{2} & K \frac{h_{2}}{2} \\
-j \kappa_{1} G_{1} A_{1} \frac{\pi}{2 L} & -j \kappa_{2} G_{2} A_{2} \frac{\pi}{2 L} & -\left(\kappa G A_{0}-P_{\mathrm{cr}}^{*}\right) \frac{\pi^{2}}{4 L^{2}} & 0 & 0 \\
-K \frac{h_{1}}{2} & -K \frac{h_{2}}{2} & 0 & K+E_{1} A_{1} \frac{\pi^{2}}{4 L^{2}} & -K \\
K \frac{h_{1}}{2} & K \frac{h_{2}}{2} & 0 & -K & K+E_{2} A_{2} \frac{\pi^{2}}{4 L^{2}}
\end{array}\right|=0
$$

Box III.

$$
\left|\begin{array}{ccccc}
-E I_{0} D^{2}+\kappa G A_{0}+K \frac{h_{0}^{2}}{2} & K \frac{h_{0}^{2}}{2} & -\kappa G A_{0} D & -K h_{0} & K h_{0} \\
K \frac{h_{0}^{2}}{2} & -E I_{0} D^{2}+\kappa G A_{0}+K \frac{h_{0}^{2}}{2} & -\kappa G A_{0} D & -K h_{0} & K h_{0} \\
-\kappa G A_{0} D & -\kappa G A_{0} D & 2\left(\kappa G A_{0}-P\right) D^{2} & 0 & 0 \\
-K h_{0} & -K h_{0} & 0 & 2 K-E A_{0} D^{2} & -2 K \\
K h_{0} & K h_{0} & 0 & -2 K & 2 K-E A_{0} D^{2}
\end{array}\right|[X]=0
$$

Box IV.

\section{Haringx-Timoshenko theory for partial composite beam-columns-differential shear model}

\subsection{Variational formulation for the buckling problem}

The same kind of study as conducted in Section 9 using the Engesser model, will now be addressed using the Haringx model. Schnabl and Planinc [8] recently considered a similar Haringx based partial composite Timoshenko beam-column model including the additional extensibility effect according to the Reissner model $[47,46]$. Proceeding as in Section 9 for the clamped-free case, the energy functional based on Haringx model is given by

$$
\begin{aligned}
& U\left[u_{1}, u_{2}, w, \psi_{1}, \psi_{2}\right] \\
& =\int_{0}^{L}\left[\frac{1}{2} E_{1} I_{1} \psi_{1}^{\prime 2}+\frac{1}{2}\left(\kappa_{1} G_{1} A_{1}-\overline{N_{1}}\right)\left(w^{\prime}-\psi_{1}\right)^{2}\right. \\
& \left.\quad+\frac{1}{2} \overline{N_{1}} w^{\prime 2}+\overline{N_{1}} u_{1}^{\prime}\right] \mathrm{d} x+\int_{0}^{L}\left[\frac{1}{2} E_{2} I_{2} \psi_{2}^{\prime 2}\right. \\
& \quad+\frac{1}{2}\left(\kappa_{2} G_{2} A_{2}-\overline{N_{2}}\right)\left(w^{\prime}-\psi_{2}\right)^{2} \\
& \left.\quad+\frac{1}{2} \overline{N_{2}} w^{\prime 2}+\overline{N_{2}} u_{2}^{\prime}+\frac{1}{2} K \cdot \Delta u^{2}\right] \mathrm{d} x \\
& \quad+P_{1} u_{1}(L)+P_{2} u_{2}(L)-\int_{0}^{L} q w \mathrm{~d} x
\end{aligned}
$$

where the slip is given by Eq. (90b). For uniform shear $\left(\psi_{1}=\psi_{2}\right)$, Eq. (105) is reduced to Eq. (80). In a corresponding way as before, the following system of differential equations is obtained

$$
\left\{\begin{array}{l}
-E_{1} I_{1} \psi_{1}^{\prime \prime}-\left(\kappa_{1} G_{1} A_{1}-\overline{N_{1}}\right)\left(w^{\prime}-\psi_{1}\right) \\
\quad+K \frac{h_{1}}{2}\left(u_{2}-u_{1}+\frac{h_{1}}{2} \psi_{1}+\frac{h_{2}}{2} \psi_{2}\right)=0 \\
-E_{2} I_{2} \psi_{2}^{\prime \prime}-\left(\kappa_{2} G_{2} A_{2}-\overline{N_{2}}\right)\left(w^{\prime}-\psi_{2}\right) \\
\quad+K \frac{h_{2}}{2}\left(u_{2}-u_{1}+\frac{h_{1}}{2} \psi_{1}+\frac{h_{2}}{2} \psi_{2}\right)=0 \\
-\left[\left(\kappa_{1} G_{1} A_{1}-\overline{N_{1}}\right)\left(w^{\prime}-\psi_{1}\right)\right]^{\prime} \\
\quad-\left[\left(\kappa_{2} G_{2} A_{2}-\overline{N_{2}}\right)\left(w^{\prime}-\psi_{2}\right)\right]^{\prime}-\left[\left(\overline{N_{1}}+\overline{N_{2}}\right) w^{\prime}\right]^{\prime}-q=0 \\
-\left(\overline{N_{1}}\right)^{\prime}-K\left(u_{2}-u_{1}+\frac{h_{1}}{2} \psi_{1}+\frac{h_{2}}{2} \psi_{2}\right)=0 \\
-\left(\overline{N_{2}}\right)^{\prime}+K\left(u_{2}-u_{1}+\frac{h_{1}}{2} \psi_{1}+\frac{h_{2}}{2} \psi_{2}\right)=0
\end{array}\right.
$$

with the following boundary conditions:

$$
\begin{aligned}
& {\left[E_{1} I_{1} \psi_{1}^{\prime} \delta \psi_{1}\right]_{0}^{L}=0} \\
& {\left[E_{2} I_{2} \psi_{2}^{\prime} \delta \psi_{2}\right]_{0}^{L}=0} \\
& \quad\left[\left(\left(\kappa_{1} G_{1} A_{1}-\overline{N_{1}}\right)\left(w^{\prime}-\psi_{1}\right)+\left(\kappa_{2} G_{2} A_{2}-\overline{N_{2}}\right)\left(w^{\prime}-\psi_{2}\right)\right.\right. \\
& \left.\left.\quad+\left(\overline{N_{1}}+\overline{N_{2}}\right) w^{\prime}\right) \delta w\right]_{0}^{L}=0 \\
& {\left[\overline{N_{1}} \delta u_{1}\right]_{0}^{L}+P_{1} \delta u_{1}(L)=0} \\
& {\left[\overline{N_{2}} \delta u_{2}\right]_{0}^{L}+P_{2} \delta u_{2}(L)=0 .}
\end{aligned}
$$


The fourth and the fifth differential equations of Eq. (106) lead to the same simplification as given by Eq. (30). The system of differential equations is then reformulated as:

$$
\left\{\begin{array}{c}
-E_{1} I_{1} \psi_{1}^{\prime \prime}-\left(\kappa_{1} G_{1} A_{1}-\overline{N_{1}}\right)\left(w^{\prime}-\psi_{1}\right) \\
\quad+K \frac{h_{1}}{2}\left(u_{2}-u_{1}+\frac{h_{1}}{2} \psi_{1}+\frac{h_{2}}{2} \psi_{2}\right)=0 \\
-E_{2} I_{2} \psi_{2}^{\prime \prime}-\left(\kappa_{2} G_{2} A_{2}-\overline{N_{2}}\right)\left(w^{\prime}-\psi_{2}\right) \\
\quad+K \frac{h_{2}}{2}\left(u_{2}-u_{1}+\frac{h_{1}}{2} \psi_{1}+\frac{h_{2}}{2} \psi_{2}\right)=0 \\
-\left[\left(\kappa_{1} G_{1} A_{1}-\overline{N_{1}}\right)\left(w^{\prime}-\psi_{1}\right)\right]^{\prime} \\
\quad-\left[\left(\kappa_{2} G_{2} A_{2}-\overline{N_{2}}\right)\left(w^{\prime}-\psi_{2}\right)\right]^{\prime} \\
\quad+P w^{\prime \prime}-q=0 \\
-E_{1} A_{1} u_{1}^{\prime \prime}-K\left(u_{2}-u_{1}+\frac{h_{1}}{2} \psi_{1}+\frac{h_{2}}{2} \psi_{2}\right)=0 \\
-E_{2} A_{2} u_{2}^{\prime \prime}+K\left(u_{2}-u_{1}+\frac{h_{1}}{2} \psi_{1}+\frac{h_{2}}{2} \psi_{2}\right)=0
\end{array}\right.
$$

with the following boundary conditions:

$$
\begin{aligned}
& {\left[E_{1} I_{1} \psi_{1}^{\prime} \delta \psi_{1}\right]_{0}^{L}=0} \\
& {\left[E_{2} I_{2} \psi_{2}^{\prime} \delta \psi_{2}\right]_{0}^{L}=0} \\
& {\left[\left(\kappa G A_{0} w^{\prime}-\left(\kappa_{1} G_{1} A_{1}-\overline{N_{1}}\right) \psi_{1}\right.\right.} \\
& \left.\left.\quad-\left(\kappa_{2} G_{2} A_{2}-\overline{N_{2}}\right) \psi_{2}\right) \delta w\right]_{0}^{L}=0 \\
& u_{1}(0)=0 ; \quad E_{1} A_{1} u_{1}^{\prime}(L)=-P_{1} \\
& u_{2}(0)=0 ; \quad E_{2} A_{2} u_{2}^{\prime}(L)=-P_{2} .
\end{aligned}
$$

Using the first and the second differential equations of (108), the system of differential equations Eq. (108) can be reformulated as:

$$
\left\{\begin{array}{c}
-E_{1} I_{1} \psi_{1}^{\prime \prime}-\left(\kappa_{1} G_{1} A_{1}-\overline{N_{1}}\right)\left(w^{\prime}-\psi_{1}\right) \\
\quad+K \frac{h_{1}}{2}\left(u_{2}-u_{1}+\frac{h_{1}}{2} \psi_{1}+\frac{h_{2}}{2} \psi_{2}\right)=0 \\
-E_{2} I_{2} \psi_{2}^{\prime \prime}-\left(\kappa_{2} G_{2} A_{2}-\overline{N_{2}}\right)\left(w^{\prime}-\psi_{2}\right) \\
\quad+K \frac{h_{2}}{2}\left(u_{2}-u_{1}+\frac{h_{1}}{2} \psi_{1}+\frac{h_{2}}{2} \psi_{2}\right)=0 \\
E_{1} I_{1} \psi_{1}^{\prime \prime \prime}+E_{2} I_{2} \psi_{2}^{\prime \prime \prime} \\
\quad-K h_{0}\left(u_{2}^{\prime}-u_{1}^{\prime}+\frac{h_{1}}{2} \psi_{1}^{\prime}+\frac{h_{2}}{2} \psi_{2}^{\prime}\right) \\
\quad+P w^{\prime \prime}-q=0 \\
-E_{1} A_{1} u_{1}^{\prime \prime}-K\left(u_{2}-u_{1}+\frac{h_{1}}{2} \psi_{1}+\frac{h_{2}}{2} \psi_{2}\right)=0 \\
-E_{2} A_{2} u_{2}^{\prime \prime}+K\left(u_{2}-u_{1}+\frac{h_{1}}{2} \psi_{1}+\frac{h_{2}}{2} \psi_{2}\right)=0 .
\end{array}\right.
$$

This system of differential equations can be linearized as:

$$
\left\{\begin{array}{l}
-E_{1} I_{1} \psi_{1}^{\prime \prime}-\left(\kappa_{1} G_{1} A_{1}+P_{1}\right)\left(w^{\prime}-\psi_{1}\right) \\
+K \frac{h_{1}}{2}\left(u_{2}-u_{1}+\frac{h_{1}}{2} \psi_{1}+\frac{h_{2}}{2} \psi_{2}\right)=0 \\
-E_{2} I_{2} \psi_{2}^{\prime \prime}-\left(\kappa_{2} G_{2} A_{2}+P_{2}\right)\left(w^{\prime}-\psi_{2}\right) \\
\quad+K \frac{h_{2}}{2}\left(u_{2}-u_{1}+\frac{h_{1}}{2} \psi_{1}+\frac{h_{2}}{2} \psi_{2}\right)=0 \\
E_{1} I_{1} \psi_{1}^{\prime \prime \prime}+E_{2} I_{2} \psi_{2}^{\prime \prime \prime}-K h_{0}\left(u_{2}^{\prime}-u_{1}^{\prime}+\frac{h_{1}}{2} \psi_{1}^{\prime}+\frac{h_{2}}{2} \psi_{2}^{\prime}\right) \\
\quad+P w^{\prime \prime}-q=0 \\
-E_{1} A_{1} u_{1}^{\prime \prime}-K\left(u_{2}-u_{1}+\frac{h_{1}}{2} \psi_{1}+\frac{h_{2}}{2} \psi_{2}\right)=0 \\
-E_{2} A_{2} u_{2}^{\prime \prime}+K\left(u_{2}-u_{1}+\frac{h_{1}}{2} \psi_{1}+\frac{h_{2}}{2} \psi_{2}\right)=0
\end{array}\right.
$$

with the following boundary conditions:

$\left[E_{1} I_{1} \psi_{1}^{\prime} \delta \psi_{1}\right]_{0}^{L}=0$
$\left[E_{2} I_{2} \psi_{2}^{\prime} \delta \psi_{2}\right]_{0}^{L}=0$

$\left[\left(\kappa G A_{0} w^{\prime}-\left(\kappa_{1} G_{1} A_{1}+P_{1}\right) \psi_{1}\right.\right.$

$$
\left.\left.-\left(\kappa_{2} G_{2} A_{2}+P_{2}\right) \psi_{2}\right) \delta w\right]_{0}^{L}=0
$$

$u_{1}(0)=0 ; \quad E_{1} A_{1} u_{1}^{\prime}(L)=-P_{1}$

$u_{2}(0)=0 ; \quad E_{2} A_{2} u_{2}^{\prime}(L)=-P_{2}$.

10.2. Simplified buckling solution for a double symmetrical crosssection

The same case of two identical sub-elements will first be treated as in Section 9.2 with the geometrical and material parameters according to Eq. (95). Subtracting the first and the second differential equations in Eq. (111) leads to the following second-order differential equation:

$$
\begin{aligned}
& E I_{0}\left(\psi_{1}-\psi_{2}\right)^{\prime \prime}-\left(\kappa G A_{0}+P\right)\left(\psi_{1}-\psi_{2}\right)=0 \text { with } \\
& P_{1}=P_{2}=\frac{P}{2}
\end{aligned}
$$

with the boundary conditions for each sub-element beam-column expressed as in Eq. (97) and then condensed as in Eq. (98) for symmetrical boundary conditions. As for the Engesser differential shear model, the uniform solution (99) is also a solution for the corresponding Haringx model in the symmetrical case. This leads to the same Haringx buckling load as given by Eq. (87).

\subsection{Buckling solution for a general cross-section}

The differential system in Eq. (106) or, equivalently, the linearized system of Eq. (111), can be presented in a differential matrix format (see Eq. (114) in Box V), where $D=\mathrm{d} / \mathrm{d} x$ is the differential operator. Proceeding as in Section 9.3, the buckling solution is found from Eq. (115) given in Box VI. The differential equations (115) can be combined into a single tenthorder differential equation satisfied by $X=\psi_{1}, \psi_{2}, w, u_{1}$ or $u_{2}$. For the fixed-free column the buckling equation is obtained (see Eq. (116) in Box VII) by inserting Eq. (54) into Eq. (115). For the same example as in Section 9.3, the numerical buckling solutions for Eq. (116) are summarized in Table 2 together with the other composite buckling models. The difference between the Engesser and Haringx models is negligible for this case.

In the double symmetrical case, the determinant (115) can be written as Eq. (117) (see Box VIII). As before, this tenth-order differential equation can be factorized to give

$$
\begin{aligned}
& E A_{0} D^{2}\left(E I_{0} D^{2}-\kappa G A_{0}-P\right)\left[\frac{\kappa G A_{0}}{\kappa G A_{0}+P} D^{6}\right. \\
& \left.+\left[\frac{P}{E I_{0}}-\alpha^{2} \frac{\kappa G A_{0}}{\kappa G A_{0}+P}\right] D^{4}-\alpha^{2} \frac{P}{E I_{\infty}} D^{2}\right][X]=0 \\
& \quad \text { with } \alpha^{2}=K\left(\frac{h_{0}^{2}}{E I_{0}}+\frac{4}{E A_{0}}\right)=K \frac{4 E I_{\infty}}{E A_{0} E I_{0}} .
\end{aligned}
$$

For the deflection function $X=w$, Eq. (118) is reduced to Eq. (85).

The same comments and conclusions for the Engesser model as given in the last two paragraphs in Section 9.3 are valid also for the Haringx model.

\section{Engesser and Haringx differential shear models for partial composite beam-columns-different boundary conditions}

This paper has been mainly focused on the study of composite beam-columns with clamped-free boundary conditions, where the following buckling mode has been assumed 


\begin{tabular}{|c}
$\left(\begin{array}{ccccc|}-E_{1} I_{1} D^{2}+\kappa_{1} G_{1} A_{1}+P_{1}+K \frac{h_{1}^{2}}{4} & K \frac{h_{1} h_{2}}{4} & -\left(\kappa_{1} G_{1} A_{1}+P_{1}\right) D & -K \frac{h_{1}}{2} & K \frac{h_{1}}{2} \\
K \frac{h_{1} h_{2}}{4} & -E_{2} I_{2} D^{2}+\kappa_{2} G_{2} A_{2}+P_{2}+K \frac{h_{2}^{2}}{4} & -\left(\kappa_{2} G_{2} A_{2}+P_{2}\right) D & -K \frac{h_{2}}{2} & K \frac{h_{2}}{2} \\
-\left(\kappa_{1} G_{1} A_{1}+P_{1}\right) D & -\left(\kappa_{2} G_{2} A_{2}+P_{2}\right) D & \kappa G A_{0} D^{2} & 0 & 0 \\
-K \frac{h_{1}}{2} & -K \frac{h_{2}}{2} & 0 & K-E_{1} A_{1} D^{2} & -K \\
K \frac{h_{1}}{2} & K \frac{h_{2}}{2} & 0 & -K & K-E_{2} A_{2} D^{2}\end{array}\right)$ \\
$\times\left(\begin{array}{l}\psi_{1} \\
\psi_{2} \\
w \\
u_{1} \\
u_{2}\end{array}\right)=\left(\begin{array}{l}0 \\
0 \\
q \\
0 \\
0\end{array}\right)$ \\
\hline
\end{tabular}

Box V.

$$
\left|\begin{array}{ccccc}
-E_{1} I_{1} D^{2}+\kappa_{1} G_{1} A_{1}+P_{1}+K \frac{h_{1}^{2}}{4} & K \frac{h_{1} h_{2}}{4} & -\left(\kappa_{1} G_{1} A_{1}+P_{1}\right) D & -K \frac{h_{1}}{2} & K \frac{h_{1}}{2} \\
K \frac{h_{1} h_{2}}{4} & -E_{2} I_{2} D^{2}+\kappa_{2} G_{2} A_{2}+P_{2}+K \frac{h_{2}^{2}}{4} & -\left(\kappa_{2} G_{2} A_{2}+P_{2}\right) D & -K \frac{h_{2}}{2} & K \frac{h_{2}}{2} \\
-\left(\kappa_{1} G_{1} A_{1}+P_{1}\right) D & -\left(\kappa_{2} G_{2} A_{2}+P_{2}\right) D & \kappa G A_{0} D^{2} & 0 & 0 \\
-K \frac{h_{1}}{2} & -K \frac{h_{2}}{2} & 0 & K-E_{1} A_{1} D^{2} & -K \\
K \frac{h_{1}}{2} & K \frac{h_{2}}{2} & 0 & -K & K-E_{2} A_{2} D^{2}
\end{array}\right|[X]=0
$$

Box VI.

\begin{tabular}{|ccccc}
$E_{1} I_{1} \frac{\pi^{2}}{4 L^{2}}+\kappa_{1} G_{1} A_{1}+\frac{E_{1} A_{1}}{E A_{0}} P_{\mathrm{cr}}^{*}+K \frac{h_{1}^{2}}{4}$ & $K \frac{h_{1} h_{2}}{4}$ & $-j\left(\kappa_{1} G_{1} A_{1}+\frac{E_{1} A_{1}}{E A_{0}} P_{\mathrm{cr}}^{*}\right) \frac{\pi}{2 L}$ & $-K \frac{h_{1}}{2}$ & $K \frac{h_{1}}{2}$ \\
$K \frac{h_{1} h_{2}}{4}$ & $E_{2} I_{2} \frac{\pi^{2}}{4 L^{2}}+\kappa_{2} G_{2} A_{2}+\frac{E_{2} A_{2}}{E A_{0}} P_{\mathrm{cr}}^{*}+K \frac{h_{2}^{2}}{4}$ & $-j\left(\kappa_{2} G_{2} A_{2}+\frac{E_{2} A_{2}}{E A_{0}} P_{\mathrm{cr}}^{*}\right) \frac{\pi}{2 L}$ & $-K \frac{h_{2}}{2}$ & $K \frac{h_{2}}{2}$ \\
$-j\left(\kappa_{1} G_{1} A_{1}+\frac{E_{1} A_{1}}{E A_{0}} P_{\mathrm{cr}}^{*}\right) \frac{\pi}{2 L}$ & $-j\left(\kappa_{2} G_{2} A_{2}+\frac{E_{2} A_{2}}{E A_{0}} P_{\mathrm{cr}}^{*}\right) \frac{\pi}{2 L}$ & $-\kappa G A_{0} \frac{\pi^{2}}{4 L^{2}}$ & 0 & 0 \\
$-K \frac{h_{1}}{2}$ & $-K \frac{h_{2}}{2}$ & 0 & $K+E_{1} A_{1} \frac{\pi^{2}}{4 L^{2}}$ & $-K$ \\
$K \frac{h_{1}}{2}$ & $K \frac{h_{2}}{2}$ & 0 & $-K$ & $K+E_{2} A_{2} \frac{\pi^{2}}{4 L^{2}}$
\end{tabular} \mid

Box VII.

\begin{tabular}{|ccccc|}
\hline$-E I_{0} D^{2}+\kappa G A_{0}+P+K \frac{h_{0}^{2}}{2}$ & $K \frac{h_{0}^{2}}{2}$ & $-\left(\kappa G A_{0}+P\right) D$ & $-K h_{0}$ & $K h_{0}$ \\
$K \frac{h_{0}^{2}}{2}$ & $-E I_{0} D^{2}+\kappa G A_{0}+P+K \frac{h_{0}^{2}}{2}$ & $-\left(\kappa G A_{0}+P\right) D$ & $-K h_{0}$ & $K h_{0}$ \\
$-\left(\kappa G A_{0}+P\right) D$ & $-\left(\kappa G A_{0}+P\right) D$ & $2 \kappa G A_{0} D^{2}$ & 0 & 0 \\
$-K h_{0}$ & $-K h_{0}$ & 0 & $2 K-E A_{0} D^{2}$ & $-2 K$ \\
$K h_{0}$ & $K h_{0}$ & 0 & $-2 K$ & $2 K-E A_{0} D^{2}$
\end{tabular} \mid$[X]=0 \quad$ (117)

Box VIII.

$w=w_{0}\left[\cos \left(\frac{\pi x}{2 L}\right)-1\right]$

$u_{i}=-\frac{P}{E A_{0}} x+u_{i, 0} \sin \left(\frac{\pi x}{2 L}\right)$;

$\psi_{i}=\psi_{i, 0} \sin \left(\frac{\pi x}{2 L}\right) \quad$ for $i \in\{1 ; 2\}$

where $P / E A_{0}=P_{i} / E_{i} A_{i}$. These equations satisfy all boundary conditions for the clamped-free problem, for example, the axial boundary conditions $u_{i}(0)=0$ and $u_{i}^{\prime}(L)=-P / E A_{0}$ satisfy Eqs. (44c) and (44d). Therefore, one can argue that the analytical buckling solutions derived in this paper are the exact ones.
In this section we will extend the study to treat other boundary conditions as hinged-hinged and clamped-clamped boundary conditions. Primarily, the differential shear models according to Engesser and Haringx discussed in Sections 9 and 10 will be the basis for the analyses.

\subsection{Hinged-hinged boundary conditions}

For the double symmetrical cross-section treated in Section 9.2 where the differential shear model gives the same result as the uniform model the Engesser generalized formula (79) for 
Table 3

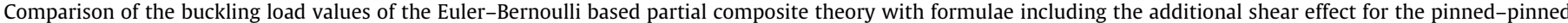
column. Numerical data according to Eq. (70) and $E_{1} / G_{1}=16$ and $E_{2} / G_{2}=16$. (Comparison with the numerical results of Schnabl and Planinc [8].)

\begin{tabular}{|c|c|c|c|c|c|c|}
\hline$K\left(\mathrm{kN} / \mathrm{cm}^{2}\right)$ & $\begin{array}{l}P_{\mathrm{cr}}(\mathrm{N}) \\
\text { Euler-Bernoulli }\end{array}$ & $\begin{array}{l}P_{\mathrm{cr}}^{*}(\mathrm{~N}) \text { Engesser } \\
\psi_{1}=\psi_{2}\end{array}$ & $\begin{array}{l}P_{\mathrm{cr}}^{*}(\mathrm{~N}) \text { Haringx } \\
\psi_{1}=\psi_{2}\end{array}$ & $\begin{array}{l}P_{\mathrm{cr}}^{*}(\mathrm{~N}) \text { Engesser } \\
\psi_{1} \neq \psi_{2}\end{array}$ & $\begin{array}{l}P_{\mathrm{cr}}^{*}(\mathrm{~N}) \text { Haringx } \\
\psi_{1} \neq \psi_{2}\end{array}$ & $\begin{array}{l}P_{\mathrm{cr}}^{*}(\mathrm{~N}) \text { Schnabl and } \\
\text { Planinc }[8]\end{array}$ \\
\hline $10^{-10}$ & 92547.541 & 91847.666 & 91852.589 & 90963.042 & 90974.110 & 90974.109 \\
\hline $10^{-5}$ & 92528.541 & 91848.652 & 91853.575 & 90964.008 & 90975.077 & 90975.077 \\
\hline $10^{-3}$ & 92627.505 & 91946.166 & 91951.105 & 91059.652 & 91070.755 & 91070.755 \\
\hline $10^{-2}$ & 93523.952 & 92829.410 & 92834.492 & 91925.866 & 91937.289 & 91937.288 \\
\hline $10^{-1}$ & 102179.815 & 101351.330 & 101357.941 & 100275.239 & 100290.056 & 100290.05 \\
\hline 1 & 166043.272 & 163866.557 & 163894.363 & 161071.853 & 161132.997 & 161132.99 \\
\hline 10 & 309799.300 & 302306.942 & 302479.779 & 292930.496 & 293295.119 & 293295.11 \\
\hline 100 & 362613.060 & 352390.547 & 352663.346 & 339715.054 & 340282.186 & 340282.18 \\
\hline 1000 & 369341.777 & 358741.907 & 359029.597 & 345613.890 & 346210.876 & 346210.87 \\
\hline $10^{5}$ & 370102.460 & 359459.512 & 359748.917 & 346279.886 & 346880.307 & 346880.32 \\
\hline $10^{10}$ & 370110.165 & 359466.780 & 359756.203 & 346286.631 & 346887.087 & 346887.09 \\
\hline
\end{tabular}

Table 4

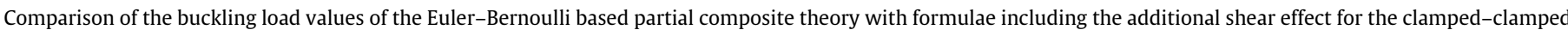
column. Numerical data according to Eq. (70) and $E_{1} / G_{1}=16$ and $E_{2} / G_{2}=16$. (Comparison with the numerical results of Schnabl and Planinc [8].)

\begin{tabular}{|c|c|c|c|c|c|c|}
\hline$K\left(\mathrm{kN} / \mathrm{cm}^{2}\right)$ & $\begin{array}{l}P_{\mathrm{cr}}(\mathrm{N}) \\
\text { Euler-Bernoulli }\end{array}$ & $\begin{array}{l}P_{\mathrm{cr}}^{*}(\mathrm{~N}) \text { Engesser } \\
\psi_{1}=\psi_{2}\end{array}$ & $\begin{array}{l}P_{\mathrm{cr}}^{*}(\mathrm{~N}) \text { Haringx } \\
\psi_{1}=\psi_{2}\end{array}$ & $\begin{array}{l}P_{\mathrm{cr}}^{*}(\mathrm{~N}) \text { Engesser } \\
\psi_{1} \neq \psi_{2}\end{array}$ & $\begin{array}{l}P_{\mathrm{cr}}^{*}(\mathrm{~N}) \text { Haringx } \\
\psi_{1} \neq \psi_{2}\end{array}$ & $\begin{array}{l}P_{\mathrm{cr}}^{*}(\mathrm{~N}) \text { Schnabl and } \\
\text { Planinc }[8]\end{array}$ \\
\hline $10^{-10}$ & 370110.165 & 359466.780 & 359756.203 & 346560.695 & 347148.125 & 347148.12 \\
\hline $10^{-5}$ & 370111.165 & 359467.724 & 359757.149 & 346561.579 & 347149.006 & 347149.00 \\
\hline $10^{-3}$ & 370210.156 & 359561.102 & 359850.751 & 346648.365 & 347236.237 & 347236.22 \\
\hline $10^{-2}$ & 371109.265 & 360409.171 & 360700.857 & 347436.547 & 348028.413 & 348026.84 \\
\hline $10^{-1}$ & 380020.906 & 368808.510 & 369120.887 & 355235.572 & 355867.913 & 355728.59 \\
\hline 1 & 461847.959 & 445391.699 & 445939.029 & 425746.792 & 426831.136 & 420203.27 \\
\hline 10 & 896250.738 & 836288.783 & 839826.107 & 769610.596 & 775920.367 & 703391.31 \\
\hline 100 & 1369477.822 & 1234255.030 & 1245396.772 & 1094325.923 & 1112314.858 & 1008246.6 \\
\hline 1000 & 1468247.704 & 1313915.438 & 1327307.985 & 1156492.916 & 1177704.260 & 1132268.7 \\
\hline $10^{5}$ & 1480317.390 & 1323572.768 & 1337256.908 & 1163968.169 & 1185591.227 & 1180748.5 \\
\hline $10^{10}$ & 1480440.659 & 1323671.313 & 1337358.449 & 1164044.380 & 1185671.662 & 1185185 \\
\hline
\end{tabular}

the buckling load can be used for composite beam-columns with interlayer slip. Correspondingly, for the Haringx model, the generalized formula (87) can be used.

For a general cross-section, the hinged-hinged buckling load for the Engesser model is obtained from the determinant given in Box IX and for the Haringx model from Eq. (121) given in Box X. The buckling solution of Eq. (120) or (121) are obtained using the following buckling shape satisfying the boundary conditions:

$$
\begin{aligned}
& w=w_{0} \sin \left(\frac{\pi x}{L}\right) ; \quad u_{i}=-\frac{P}{E A_{0}} x+u_{i, 0} \cos \left(\frac{\pi x}{L}\right) ; \\
& \psi_{i}=\psi_{i, 0} \cos \left(\frac{\pi x}{L}\right) \quad \text { for } i \in\{1 ; 2\} .
\end{aligned}
$$

In particular, the axial boundary conditions for this hinged-hinged case are given by

$E_{i} A_{i} u_{i}^{\prime}(0)=-P_{i} \quad$ and $\quad E_{i} A_{i} u_{i}^{\prime}(L)=-P_{i} \quad$ for $i \in\{1 ; 2\}$.

These axial boundary conditions are symmetrical with respect to the axial loads. They correspond, for instance, to a symmetrical loading mode associated with a symmetrical constraint. The numerical results based on the Eqs. (120)-(121) are presented in Table 3 with the shear parameters $E_{1} / G_{1}=E_{2} / G_{2}=16$, the same as those chosen by Schnabl and Planinc [8] in a recent paper. We have compared our Haringx based differential shear results with the numerical results of Schnabl and Planinc [8] neglecting the additional extensibility effect and we found close agreement up to 4 digits. For this case, the differential shear Haringx model as presented in this paper and the one based on the Reissner model are strictly equivalent.

\subsection{Clamped-clamped boundary conditions}

For the double symmetrical cross-section treated in Section 9.2 where the differential shear model gives the same result as the uniform model the Engesser generalized formula (79) for the buckling load can be used for composite beam-columns with interlayer slip. Correspondingly, for the Haringx model, the generalized formula (87) can be used.

For a general cross-section, the clamped-clamped buckling load for the Engesser model is obtained from the determinant given in Box XI and for the Haringx model from Eq. (125) given in Box XII.

The buckling solution of Eq. (124) or (125) are obtained using the following buckling shape, satisfying the boundary conditions:

$$
\begin{aligned}
& w=w_{0}\left[\cos \left(\frac{2 \pi x}{L}\right)-1\right] \\
& u_{i}=-\frac{P}{E A_{0}} x+u_{i, 0} \sin \left(\frac{2 \pi x}{L}\right) ; \\
& \psi_{i}=\psi_{i, 0} \sin \left(\frac{2 \pi x}{L}\right) \quad \text { for } i \in\{1 ; 2\} .
\end{aligned}
$$

The axial boundary conditions for this clamped-clamped case are given by

$u_{i}(0)=0 \quad$ and $\quad u_{i}(L)=-P L / E A_{0} \quad$ for $i \in\{1 ; 2\}$.

The numerical results are presented in Table 4. It is evident that the effect of the shear model is more pronounced for clamped-clamped boundary conditions than, for example, for clamped-free boundary conditions. We also noticed a small difference between the results presented in this paper, and the results published by Schnabl and Planinc [8] for the clamped-clamped boundary conditions. The reason for this difference is due to the difference in modelling the axial boundary conditions (see also the discussion of Schnabl and Planinc [7] on this specific effect). In particular, the axial boundary conditions modelled in this paper for the clamped-clamped boundary condition according to Eq. (126) do not fulfil all the axial boundary conditions:

$E_{i} A_{i} u_{i}^{\prime}(L) \neq-P_{i} \quad$ for $i \in\{1 ; 2\}$. 


$$
\left|\begin{array}{ccccc}
E_{1} I_{1} \frac{\pi^{2}}{L^{2}}+\kappa_{1} G_{1} A_{1}+K \frac{h_{1}^{2}}{4} & K \frac{h_{1} h_{2}}{4} & -j \kappa_{1} G_{1} A_{1} \frac{\pi}{L} & -K \frac{h_{1}}{2} & K \frac{h_{1}}{2} \\
K \frac{h_{1} h_{2}}{4} & E_{2} I_{2} \frac{\pi^{2}}{L^{2}}+\kappa_{2} G_{2} A_{2}+K \frac{h_{2}^{2}}{4} & -j \kappa_{2} G_{2} A_{2} \frac{\pi}{L} & -K \frac{h_{2}}{2} & K \frac{h_{2}}{2} \\
-j \kappa_{1} G_{1} A_{1} \frac{\pi}{L} & -j \kappa_{2} G_{2} A_{2} \frac{\pi}{L} & -\left(\kappa G A_{0}-P_{\mathrm{cr}}^{*}\right) \frac{\pi^{2}}{L^{2}} & 0 & 0 \\
-K \frac{h_{1}}{2} & -K \frac{h_{2}}{2} & 0 & K+E_{1} A_{1} \frac{\pi^{2}}{L^{2}} & -K \\
K \frac{h_{1}}{2} & K \frac{h_{2}}{2} & 0 & -K & K+E_{2} A_{2} \frac{\pi^{2}}{L^{2}}
\end{array}\right|=0
$$

Box IX.

$$
\left|\begin{array}{ccccc}
E_{1} I_{1} \frac{\pi^{2}}{L^{2}}+\kappa_{1} G_{1} A_{1}+\frac{E_{1} A_{1}}{E A_{0}} P_{\mathrm{cr}}^{*}+K \frac{h_{1}^{2}}{4} & K \frac{h_{1} h_{2}}{4} & -j\left(\kappa_{1} G_{1} A_{1}+\frac{E_{1} A_{1}}{E A_{0}} P_{\mathrm{cr}}^{*}\right) \frac{\pi}{L} & -K \frac{h_{1}}{2} & K \frac{h_{1}}{2} \\
K \frac{h_{1} h_{2}}{4} & E_{2} I_{2} \frac{\pi^{2}}{L^{2}}+\kappa_{2} G_{2} A_{2}+\frac{E_{2} A_{2}}{E A_{0}} P_{\mathrm{cr}}^{*}+K \frac{h_{2}^{2}}{4} & -j\left(\kappa_{2} G_{2} A_{2}+\frac{E_{2} A_{2}}{E A_{0}} P_{\mathrm{cr}}^{*}\right) \frac{\pi}{L} & -K \frac{h_{2}}{2} & K \frac{h_{2}}{2} \\
-j\left(\kappa_{1} G_{1} A_{1}+\frac{E_{1} A_{1}}{E A_{0}} P_{\mathrm{cr}}^{*}\right) \frac{\pi}{L} & -j\left(\kappa_{2} G_{2} A_{2}+\frac{E_{2} A_{2}}{E A_{0}} P_{\mathrm{cr}}^{*}\right) \frac{\pi}{L} & -\kappa A_{0} \frac{\pi^{2}}{L^{2}} & 0 & 0 \\
-K \frac{h_{1}}{2} & -K \frac{h_{2}}{2} & 0 & K+E_{1} A_{1} \frac{\pi^{2}}{L^{2}} & -K \\
K \frac{h_{1}}{2} & K \frac{h_{2}}{2} & 0 & -K & K+E_{2} A_{2} \frac{\pi^{2}}{L^{2}}
\end{array}\right|=0
$$

Box X.

$$
\left|\begin{array}{ccccc}
E_{1} I_{1} \frac{4 \pi^{2}}{L^{2}}+\kappa_{1} G_{1} A_{1}+K \frac{h_{1}^{2}}{4} & K \frac{h_{1} h_{2}}{4} & -j \kappa_{1} G_{1} A_{1} \frac{2 \pi}{L} & -K \frac{h_{1}}{2} & K \frac{h_{1}}{2} \\
K \frac{h_{1} h_{2}}{4} & E_{2} I_{2} \frac{4 \pi^{2}}{L^{2}}+\kappa_{2} G_{2} A_{2}+K \frac{h_{2}^{2}}{4} & -j \kappa_{2} G_{2} A_{2} \frac{2 \pi}{L} & -K \frac{h_{2}}{2} & K \frac{h_{2}}{2} \\
-j \kappa_{1} G_{1} A_{1} \frac{2 \pi}{L} & -j \kappa_{2} G_{2} A_{2} \frac{2 \pi}{L} & -\left(\kappa G A_{0}-P_{\mathrm{cr}}^{*}\right) \frac{4 \pi^{2}}{L^{2}} & 0 & 0 \\
-K \frac{h_{1}}{2} & -K \frac{h_{2}}{2} & 0 & K+E_{1} A_{1} \frac{4 \pi^{2}}{L^{2}} & -K \\
K \frac{h_{1}}{2} & K \frac{h_{2}}{2} & 0 & -K & K+E_{2} A_{2} \frac{4 \pi^{2}}{L^{2}}
\end{array}\right|=0
$$

Box XI.

$$
\left|\begin{array}{ccccc}
E_{1} I_{1} \frac{4 \pi^{2}}{L^{2}}+\kappa_{1} G_{1} A_{1}+\frac{E_{1} A_{1}}{E A_{0}} P_{\mathrm{cr}}^{*}+K \frac{h_{1}^{2}}{4} & K \frac{h_{1} h_{2}}{4} & -j\left(\kappa_{1} G_{1} A_{1}+\frac{E_{1} A_{1}}{E A_{0}} P_{\mathrm{cr}}^{*}\right) \frac{2 \pi}{L} & -K \frac{h_{1}}{2} & K \frac{h_{1}}{2} \\
K \frac{h_{1} h_{2}}{4} & E_{2} I_{2} \frac{4 \pi^{2}}{L^{2}}+\kappa_{2} G_{2} A_{2}+\frac{E_{2} A_{2}}{E A_{0}} P_{\mathrm{cr}}^{*}+K \frac{h_{2}^{2}}{4} & -j\left(\kappa_{2} G_{2} A_{2}+\frac{E_{2} A_{2}}{E A_{0}} P_{\mathrm{cr}}^{*}\right) \frac{2 \pi}{L} & -K \frac{h_{2}}{2} & K \frac{h_{2}}{2} \\
-j\left(\kappa_{1} G_{1} A_{1}+\frac{E_{1} A_{1}}{E A_{0}} P_{\mathrm{cr}}^{*}\right) \frac{2 \pi}{L} & -j\left(\kappa_{2} G_{2} A_{2}+\frac{E_{2} A_{2}}{E A_{0}} P_{\mathrm{cr}}^{*}\right) \frac{2 \pi}{L} & -\kappa G A_{0} \frac{4 \pi^{2}}{L^{2}} & 0 & 0 \\
-K \frac{h_{1}}{2} & -K \frac{h_{2}}{2} & 0 & K+E_{1} A_{1} \frac{4 \pi^{2}}{L^{2}} & -K \\
K \frac{h_{1}}{2} & K \frac{h_{2}}{2} & 0 & -K & K+E_{2} A_{2} \frac{4 \pi^{2}}{L^{2}}
\end{array}\right|=0
$$

Box XII.

However, we also note that the kinematic axial boundary conditions of the problem $u_{i}(0)=0$ are verified by the solution of Eq. (126). As the generalized kinematic boundary conditions are fulfilled in the clamped-clamped case, the analytical solution presented in the paper leads to an upper bound of the exact solution, thanks to the Rayleigh-Ritz method (even if all the boundary conditions, including the force boundary conditions, are not fulfilled; see for instance Alfutov [35]). This fact is clearly verified by the numerical data presented in Table 4 , where the buckling load derived by Schnabl and Planinc [8] is lower than the analytical solution based on Eq. (125). It is also worth mentioning that the analytical solution developed in this paper can also serve as the exact solution of the fully clamped-clamped partial composite problem with immovable supports for thermoelastic buckling problems under homogeneous heating associated with the boundary conditions:

$u_{i}(0)=0$ and $u_{i}(L)=0$ for $i \in\{1 ; 2\}$.

\section{Summary and conclusions}

This paper is devoted to the elastic stability problem of a partial composite column with two sub-elements connected to each other via a linear constitutive law for the slip. In the first part of the paper, each sub-element is modelled by the Euler-Bernoulli beam-column theory. The basic beam-column case studied is that with clamped-free boundary conditions. A variational formulation is presented in order to derive relevant boundary conditions. 
The specific axial loading giving rise to no pre-bending effects is geometrically characterized, leading to analytical buckling loads of the composite column. We show the equivalence between the Hoff sandwich beam-column theory, the partial composite theory with interlayer slip and the Bickford-Reddy theory, from the stability point of view. We further investigate some other loading configurations, where eccentricity end moments are introduced; the behaviour is shown to correspond to the analysis of imperfect columns. The effect of axial extensibility is analytically quantified and compared with the classical solution of the problem.

In the last part of the paper, the shear effect is introduced in each sub-element through the Timoshenko theory. As a main assumption of the Timoshenko composite beam-column theory, the shear deformation is assumed to be the same in all layers (uniform shear). A variational formulation of the buckling behaviour of the partial composite column is given, both for the Engesser and the Haringx theory. A discussion about assuming different shear deformations in the different sub-elements of the composite column with interlayer slip (differential shear) concludes the paper together with a discussion on the influence of different boundary conditions. We show the possibility to include the differential shear model into the kinematics of the partial composite beam-column and we evaluate the difference between the results of the uniform and differential shear models. These two models are equivalent in the case of double symmetrical cross-sections and beam-columns with symmetrical boundary conditions. With respect to influence of different boundary conditions it is shown that the shear effect is more pronounced, for example, for the clamped-clamped case than for the clamped-free case.

Future studies could involve refinement of the model for the kinematics of the shear layer. Vertical uplift could be introduced and also a more complex interaction law, including non-local partial interaction, could be included (see for instance [66]). Some boundary layer phenomena should also appear in the buckling of these partially composite elements, as already shown in bending analysis [60].

\section{Acknowledgements}

The authors express their sincere appreciation to the Regional Council of Västerbotten, the County Administrative Board of Västerbotten and The European Union's Structural Funds-The Regional Fund for their financial support.

\section{References}

[1] Girhammar UA, Gopu VKA. Composite beam-columns with interlayer slipexact analysis. J Struct Eng 1993;119(4):1265-82.

[2] Girhammar UA, Pan DH. Exact static analysis of partially composite beams and beam-columns. Int J Mech Sci 2007;49:239-55.

[3] Möhler K. Über das Tragverhalten von Biegeträgern und Druckstäben mit zusammengesetztem Querschnitt und nachgiebigen Verbindungsmitteln. Habilitation thesis. Karlsruhe: Technichen Hochschule Friedericiana zu Karlsruhe; 1956

[4] Girhammar UA, Gopu VKA. Analysis of $P-\Delta$ effect in composite concrete/timber beam-columns. Proc Inst Civ Eng, Part 2 1991;91:39-54.

[5] Girhammar UA, Gopu VKA. Some aspects of the analysis of partially composite beam-columns. Internal report. 1993.

[6] Xu R, Wu Y. Static, dynamic, and buckling analysis of partial interaction composite members using Timoshenko's beam theory. Int J Mech Sci 2007; 49:1139-55.

[7] Schnabl S, Planinc I. The influence of boundary conditions and axial deformability on buckling behaviour of two-layer composite columns with interlayer slip. Eng Struct 2010;32:3103-11.

[8] Schnabl S, Planinc I. The effect of transverse shear deformation on the buckling of two-layer composite columns with interlayer slip. Internat J Non-Linear Mech 2011;46(3):543-53.

[9] Norris CB, Ericksen WS, Kommers WJ. Flexural rigidity of a rectangular strip of sandwhich construction-comparison between mathematical analysis and results of tests. Tech. rep. report 1505A. Madison (Wis.): Forest Prod. Lab.; 1952.
[10] Plantema FJ. Sandwhich construction. New York: John Wiley \& Sons; 1966.

[11] Allen HG. Analysis and design of structural sandwhich panels. Oxford: Pergamon Press; 1969.

[12] Hoff NJ. The analysis of structures. London (England): John Wiley and Sons 1956.

[13] Hoff NJ, Mautner SE. Bending and buckling of sandwhich beams. J Aeronaut Sci 1948; $15: 707$.

[14] Bauld NR. Dynamic stability of sandwhich columns under pulsating axial loads. AIAA J 1967;5(8):1514-6.

[15] Kollár LP. Buckling analysis of coupled shear walls by the multi-layer sandwhich column. Acta Tech Acad Sci Hung 1986;99(3-4):317-32.

[16] Attard MM, Hunt GW. Column buckling with shear deformations-a hyperelastic formulation. Internat J Solids Struct 2008;45:4322-39.

[17] Attard MM, Hunt GW. Sandwhich column buckling-a hyperelastic formulation. Internat J Solids Struct 2008;45:5540-55.

[18] Heuer R. A correspondence for the analysis of sandwhich beams with or without interlayer slip. Mech Adv Mater Struct 2004;11:425-32.

[19] Blaauwendraad J. Shear in structural stability: on the Engesser-Haringx discord. J Appl Mech 2010;77:031005.

[20] Kardomateas GA. An elasticity solution for the global buckling of sandwhich beams/wide panels with orthotropic phases. J Appl Mech 2010;77(01015): $1-7$.

[21] Adekola AO. Partial interaction between elastically connected elements of a composite beam. Internat J Solids Struct 1968;4:1125-35.

[22] Gara F, Ranzi G, Leoni G. Displacement-based formulations for composite beams with longitudinal slip and vertical uplift. Internat J Numer Methods Engrg 2006;65:1197-220.

[23] Ranzi G, Gara F, Ansourian P. General method of analysis for composite beams with longitudinal and transverse partial interaction. Comput Struct 2006;84: 2373-84.

[24] Ranzi G, Bradford MA. Composite beams with both longitudinal and transverse partial interaction subjected to elevated temperatures. Eng Struct 2007;29: 2737-50.

[25] Kroflic A, Planinc I, Saje M, Cas B. Analytical solution of two-layer beam including interlayer slip and uplift. Struct Eng Mech 2010;34(6):667-83.

[26] Kroflic A, Planinc I, Saje M, Turk G, Cas B. Nonlinear analysis of two-layer timber beams considering interlayer slip and uplift. Eng Struct 2010;32: 1617-30.

[27] Girhammar UA, Pan DH, Gustafsson A. Exact dynamic analysis of composite beams with partial interaction. Int J Mech Sci 2009;51(8):565-82.

[28] McCutcheon WJ. Method of predicting the stiffness of wood-joist floor systems with partial composite action. Tech. Rep. Research Paper FPL 289 Forest Products Laboratory, U.S. Dept. of Agriculture, Forest Service, Madison, Wisconsin, 1977.

[29] Krawczyk P, Frey F, Zielinsky AP. Large deflections of laminated beams with interlayer slips part 1: model development. Eng Comput 2007;24(1):17-32.

[30] Challamel N. On lateral-torsional vibrations of elastic composite beams with interlayer slip. J Sound Vib 2009;325:1012-22.

[31] Challamel N, Bernard F, Casandjian C. Out-of-plane behaviour of partially composite or sandwhich beams by exact and finite element methods. ThinWalled Struct 2010;48:561-80.

[32] Reddy JN. Nonlocal theories for bending, buckling and vibration of beams. Internat J Engrg Sci 2007;45:288-307.

[33] Euler L. Methodus inveniendi lineas curvas maxima minimive proprietate gaudentes Additamentum I. Lausanne (Geneva): De Curvis Elasticis; 1774.

[34] Pflüger A. Stabililätsprobleme der elastostatik. Springer-Verlag; 1964.

[35] Alfutov NA. Stability of elastic structures. Berlin: Springer-Verlag; 2000

[36] Magnusson A, Ristinmaa M, Ljung C. Behaviour of the extensible elastica solution. Internat J Solids Struct 2001;38:8441-57.

[37] Simitses GJ, Hodges DH. Fundamentals of structural stability. Burlington: Elsevier; 2006.

[38] Engesser F. Über die Knickfestigkeit gerader Stäbe. Z Archit Ing 1889;35(4): 455-62.

[39] Haringx JA. On the buckling and lateral rigidity of helical springs. Proc Konink Ned Akad Wetenschap 1942;45:533.

[40] Bažant ZP. A correlation study of formulations of incremental deformation and stability of continuous bodies. J Appl Mech 1971;38(4):919-28.

[41] Bažant ZP, Cedolin L. Stability of structures-elastic, inelastic, fracture, and damage theories. New York: Dover Publications, Inc.; 2003.

[42] Ji W, Waas AM, Bažant ZP. Errors caused by non-work-conjugate stress and strain measures and necessary corrections in finite element programs. J Appl Mech 2010;77:044504.

[43] Bažant ZP, Beghini A. Which formulation allows using a constant shear modulus for small strain-buckling of soft-core sandwhich structures? ASME, J Appl Mech 2005;72:785-7.

[44] Bažant ZP, Beghini A. Stability and finite strain of homogenized structures soft in shear: sandwhich or fiber composites, and layered bodies. Internat J Solids Struct 2006;43:1571-93.

[45] Ziegler H. Arguments for and against Engesser's buckling formulas. Ing Arch 1982;52:105-13.

[46] Reissner E. Some remarks on the problem of Euler buckling. Ing Arch 1982;52: 115-9.

[47] Reissner E. On one-dimensional finite-strain beam theory: the plane problem. Z Angew Math Phys 1972;23:795-804.

[48] Goto Y, Yoshimitshu T, Obata M. Elliptic integral solutions of plane elastica with axial and shear deformations. Internat J Solids Struct 1990;26(4):375-90.

[49] Levinson M. A new rectangular beam theory. J Sound Vib 1981;74(6):343-50. 
[50] Bickford WB. A consistent higher order beam theory. Dev Theor Appl Mech 1982;11:137-50.

[51] Reddy JN. A simple higher-order theory for laminated composite plates. J Appl Mech 1984;51:745-52.

[52] Levinson M. On Bickford's consistent higher-order beam theory. Mech Res Comm 1985;12(1):1-9.

[53] Ghugal YM, Shimpi RP. A review of refined shear deformation theories for isotropic and anisotropic laminated beams. J Reinf Plast Compos 2001;20(3): 255-72.

[54] Shi G, Voyiadjis GZ. A sixth-order theory of shear deformable beams with variational consistent boundary conditions. J Appl Mech 2011;78(021019): $1-11$.

[55] Wang CM, Reddy JN, Lee KH. Shear deformable beams and plates: relationships with classical solutions. Oxford (UK): Elsevier; 2000.

[56] Kryžanowski A, Schnabl S, Turk G, Planinc I. Exact slip-buckling analysis of two-layer composite columns. Internat J Solids Struct 2009;46:2929-38.

[57] Schnabl S, Saje M, Turk G, Planinc I. Analytical solution of two-layer beam taking into account interlayer slip and shear deformation. J Struct Eng 2007; 133(6):886-94.
[58] Krawczyk P, Rebora B. Large deflections of laminated beams with interlayer slips part 2: finite element development. Eng Comput 2007;24(1):33-51.

[59] Sokolinsky V, Frostig Y. Boundary condition effects in buckling of soft core sandwhich panels. J Eng Mech 1999;125:865-74.

[60] Challamel N, Girhammar UA. Boundary layer effect in composite beams with interlayer slip. J Aerosp Eng, ASCE 2011;24(2):199-209.

[61] Bažant ZP. Shear buckling of sandwhich, fiber composite and lattice columns, bearing and helical springs: paradox resolved. J Appl Mech 2003;70(1): 75-83.

[62] Wang CM, Wang CY, Reddy JN. Exact solutions for buckling of structural members. CRC series in computational mechanics and applied analysis. Boca Raton (FL): 2005.

[63] Timoshenko SP, Gere JM. Theory of elastic stability. McGraw-Hill; 1961.

[64] Ranzi G, Dall'Asta A, Ragni L, Zona A. A geometric nonlinear model for composite beams with partial interaction. Eng Struct 2010;32:1384-96.

[65] Cowper GR. The shear coefficient in Timoshenko's beam theory. J Appl Mech 1966;33:335-40.

[66] Challamel N, Mechab I, Elmeiche N, Krour B. Buckling of composite nonlocal or gradient connected beams. Int J Struct Stab Dyn 2011 [in press]. 